\title{
Tune modulation effects for colliding beams in the High Luminosity Large Hadron Collider
}

\author{
S. Kostoglou $\odot,{ }^{1,2,{ }^{*}}$ H. Bartosik $\odot,{ }^{1}$ Y. Papaphilippou $\odot,{ }^{1}$ G. Sterbini $\odot,{ }^{1}$ and N. Triantafyllou $\oplus^{1,3}$ \\ ${ }^{1}$ CERN, Geneva 1211, Switzerland \\ ${ }^{2}$ National Technical University of Athens, Athens 15780, Greece \\ ${ }^{3}$ University of Liverpool, Liverpool L69 3BX, United Kingdom
}

(Received 3 March 2020; accepted 2 December 2020; published 15 December 2020)

\begin{abstract}
Several transverse noise sources, such as power supply ripple, can potentially act as an important limiting mechanism for the luminosity production of the Large Hadron Collider (LHC) and its future High-Luminosity upgrade (HL-LHC) [High Luminosity Large Hadron Collider (HL-LHC): Technical Design Report V0.1, CERN-2017-007-M [http://cds.cern.ch/record/782076?ln=en]]. In the presence of non-linearities, depending on the spectral components of the power supply ripple spectrum and the nature of the source, such a mechanism can increase the tune diffusion of the particles in the distribution through the excitation of sideband resonances in the vicinity of the resonances driven by the lattice nonlinearities. For the HL-LHC, due to the reduction of the beam size in the interaction points (IP) of the high luminosity experiments (IP1 and 5), increased sensitivity to power supply ripple is anticipated for the quadrupoles of the inner triplets. The modulation that may arise from the power supply ripple will be combined with the tune modulation that intrinsically emerges from the coupling of the transverse and longitudinal plane for off-momentum particles through chromaticity. This paper aims to study the impact of tune modulation effects on the transverse beam motion resulting from the interplay between quadrupolar power supply ripple and synchro-betatron coupling in the presence of strong head-on and long-range beam-beam interactions. A power supply ripple threshold for acceptable performance is estimated with single-particle tracking simulations by investigating the impact of different modulation frequencies and amplitudes on the dynamic aperture. The excitation of sideband resonances due to the tune modulation is demonstrated with frequency maps and the higher sensitivity to specific modulation frequencies is explained. Finally, a power supply ripple spectrum consisting of several tones is considered to determine whether their presence will limit the luminosity production in the HL-LHC era.
\end{abstract}

DOI: $10.1103 /$ PhysRevAccelBeams.23.121001

\section{INTRODUCTION}

In order to optimize the performance of a high-energy particle collider such as the Large Hadron Collider (LHC) [1] and its future High-Luminosity upgrade (HL-LHC) [2], a thorough understanding of all the phenomena that can degrade the luminosity is required. A major concern for the transverse single-particle beam dynamics is the presence of power supply ripple, i.e., a periodic perturbation in the strengths of the lattice magnets, a mechanism that can impact the long term stability of the circulating particles and prove detrimental to the beam lifetime.

\footnotetext{
*sofia.kostoglou@cern.ch
}

Published by the American Physical Society under the terms of the Creative Commons Attribution 4.0 International license. Further distribution of this work must maintain attribution to the author(s) and the published article's title, journal citation, and DOI.
Harmonics of the mains power frequency $(50 \mathrm{~Hz})$ in the power supply voltage result in magnetic field perturbations through the magnet's transfer function that is expressed as [3]:

$$
\Delta B(f)=T_{B_{m} \rightarrow B_{b}} \times T_{I \rightarrow B_{m}} \times T_{V \rightarrow I} \times V(f),
$$

where $f$ is the frequency, $V(f)$ the voltage ripple, $T_{V \rightarrow I}$ the magnet's circuit impedance, $T_{I \rightarrow B_{m}}$ the transfer function from the current to the magnetic field and $T_{B_{m} \rightarrow B_{b}}$ the magnetic field seen by the beam including the shielding effect of the vacuum chamber and the beam screen.

The present paper investigates the implications of power supply ripple in the quadrupoles located at the high $\beta$-function regions of the accelerator. These magnetic field fluctuations $\Delta B$ induce a modulation in the normalized focusing strength of the quadrupoles $\Delta k$. These gradient errors lead to a modulation of the betatron tune that is equal to: 


$$
\Delta Q=\frac{1}{4 \pi} \sum_{i} \beta_{i} \Delta k_{i} l,
$$

where $\beta_{i}$ is the $\beta$-function at the location of the $i$ th perturbation and $\Delta k_{i} l=\frac{\Delta B_{i}}{B \rho}$ with $B \rho$ representing the beam rigidity. The modulation depth is defined as the maximum variation of the tune from its unperturbed value in the absence of a tune modulation. If present, this effect is combined with the tune modulation that intrinsically emerges for off-momentum particles from the coupling of the longitudinal and transverse plane through chromaticity [4].

Tune modulation effects have been reported in the past from several hadron synchrotrons and colliders including the Tevatron [5-7], the Super Proton Synchrotron (SPS) [8-10], the Relativistic Heavy Ion Collider (RHIC) [11-13] and the Hadron-Electron Ring Accelerator (HERA) [14-18]. In the SPS, the combined effect of two modulation frequencies was experimentally proven to be more severe compared to a single frequency with an equivalent modulation depth $[8,9]$. The increase of losses and emittance growth was correlated with the overlap of multiple resonances [10].

In HERA, a dependence of the particles' diffusion on the modulation frequency was shown [18]. In particular, a loss rate increase by one order of magnitude was reported when a tune modulation in the frequency range of $600 \mathrm{~Hz}$ to $1.2 \mathrm{kHz}$ was applied, an effect that was not observed for other modulation frequencies. A tune ripple feedback was successfully employed for the compensation of the power supply ripple $[15,16]$.

Previous HL-LHC studies have revealed a sensitivity of the dynamic aperture (DA) on the modulation frequency. Specifically, a DA reduction was reported for a modulation frequency at $300 \mathrm{~Hz}$ and $600 \mathrm{~Hz}$ and a modulation depth of $10^{-4}$, an effect that was not observed with other modulation frequencies. Table I summarizes the maximum modulation depth to avoid an impact on the beam performance for various accelerators as reported from previous studies. A detailed discussion on experimental observations of power supply ripple in the LHC is presented in [19].

In the presence of nonlinearities such as sextupoles, octupoles and beam-beam interactions, tune modulation effects may lead to the excitation of sideband resonances. Depending on the modulation frequency, the resonances can either overlap, leading to chaotic trajectories [23-26],

TABLE I. The critical modulation depth $\Delta Q$ as reported from previous studies in several accelerators [11,14,20-22].

\begin{tabular}{lccc}
\hline \hline Study & $\Delta Q$ & Study & $\Delta Q$ \\
\hline RHIC & $<10^{-3}$ & LHC & $<5 \times 10^{-4}$ \\
SPS & $<10^{-3}$ & HL-LHC & $<10^{-4}$ \\
HERA & $<10^{-4}$ & & \\
\hline \hline
\end{tabular}

or reach the tune footprint, thereby acting as a diffusion mechanism for the particles in the distribution in addition to the resonances driven by the lattice nonlinearities [15]. In the latter case, the existence of such resonances critically limits the available space in frequency domain for an optimized, resonance-free working point.

Subsequently, it is important to investigate whether the combination of the tune modulation induced by power supply ripple and synchro-betatron coupling will pose a limitation to the luminosity production of the HL-LHC, as well as other present and future hadron colliders such as RHIC, the Electron-Ion Collider (EIC) [27] and the Future Circular Collider (FCC) [28]. Single-particle tracking simulations are performed in the element-byelement HL-LHC lattice including chromatic sextupoles, Landau octupoles and both head-on and long-range beambeam interactions. The two tune modulation sources considered in the present paper, power supply ripple and chromatic tune modulation, are first, studied individually and then, their combined impact is estimated.

\section{A SIMPLIFIED FORMALISM OF TUNE MODULATION EFFECTS}

In the presence of a tune modulation, the variation of the tune, referred to as the instantaneous tune, as a function of the turn number $n$ is

$$
Q_{\text {inst }}(n)=Q_{0}+\Delta Q \cdot \cos \left(2 \pi Q_{m} n\right) \text {, }
$$

where $Q_{0}$ is the unperturbed tune in the absence of the tune modulation and $\Delta Q$ and $Q_{m}$ are the modulation depth and modulation tune, respectively. In the case of a tune modulation induced by power supply ripple in quadrupole magnets, the modulation depth is the maximum value of Eq. (2). In the presence of synchro-betatron coupling, the modulation tune is the synchrotron tune $\left(Q_{m}=Q_{s}\right)$ and the modulation depth is [29]:

$$
\Delta Q=Q^{\prime} \frac{\Delta p}{p},
$$

where $Q^{\prime}$ is the chromaticity and $\frac{\Delta p}{p}$ the relative momentum deviation of the particle. The modulation index is defined as:

$$
\beta_{m}=\frac{\Delta Q}{Q_{m}} .
$$

By integrating Eq. (3), the linear betatron oscillation of a particle with an action $J$ is

$$
x(n)=\sqrt{2 J \beta} \cos \left(2 \pi Q_{0} n+\beta_{m} \sin \left(2 \pi Q_{m}\right) n\right),
$$

where $\beta$ is the $\beta$-function. As in every frequency modulated signal, considering the betatron motion as the carrier and 
the power supply ripple as the modulator, the particle's trajectory is represented in time domain by a sum of sinusoidal signals, weighted by the Bessel functions of the first kind [30]. The frequency-domain representation of the betatron motion, after normalizing by the maximum amplitude, is:

$$
\begin{aligned}
|\tilde{X}(Q)|= & \sum_{p=-\infty}^{\infty} J_{k}\left(\beta_{m}\right)\left[\delta\left(Q-Q_{0}-p \cdot Q_{m}\right)\right. \\
& \left.+\delta\left(Q+Q_{0}+p \cdot Q_{m}\right)\right],
\end{aligned}
$$

where $p$ is an integer, $J_{k}$ are the Bessel functions of the first kind and $\delta$ is the Dirac function. The spectrum consists of infinite harmonics at $Q_{0} \pm p \cdot Q_{m}$ with a relative amplitude that depends on the modulation index. In particular, the $k$-order Bessel function for a modulation index $\beta_{m}$ determines the amplitude of the $k$ sideband, with $J_{0}$ representing the betatron tune. Decreasing the modulation frequency for a constant modulation depth leads to the appearance of higher-order sidebands with larger amplitudes in the spectrum. For a critical value of the modulation index $\beta_{m}^{c}$ :

$$
J_{0}\left(\beta_{m}^{c}\right)=J_{1}\left(\beta_{m}^{c}\right) \rightarrow \beta_{m}^{c}=1.41,
$$

the amplitude of the first sideband is equal to the one of the betatron tune. For $\beta_{m}=2.4$, the amplitude of the betatron peak is suppressed as $J_{0}(2.4) \approx 0$.

Non-linear fields are an important aspect of the accelerator's lattice, introduced for the correction or enhancement of the chromatic aberrations and the action-dependent detuning of the particles. A detailed discussion on the trapping of the particles while crossing high-order nonlinear resonances, which is a mechanism that can lead to particle losses, can be found in [31]. The effect of a tune modulation in a one-dimensional nonlinear Hamiltonian system has been extensively studied in the past [17,32-34]. These studies have shown that the impact of a tune modulation on the transverse motion varies, depending on the modulation tune, $Q_{m}$, the modulation depth, $\Delta Q$, and the island tune that is defined as:

$$
Q_{I}=\frac{N}{2 \pi} J_{R}^{N / 4} \sqrt{2 \alpha V_{N}}
$$

where $J_{R}$ is the resonance action, $N$ is the resonance order, $\alpha$ is the amplitude detuning coefficient and $V_{N}$ is the resonance strength. Therefore, the island tune is a representation of the resonance strength [7,35].

Through an analytical formalism, four regimes of interest were identified in the tune modulation $\left(Q_{m}, \Delta Q\right)$ parameter space [17,32-34]. Figure 1 illustrates the four regimes in the tune modulation parameter space for the sixth-order resonance $(N=6)$.

First, a modulation at low amplitude and low frequency causes a time variation of the particles' actions, without

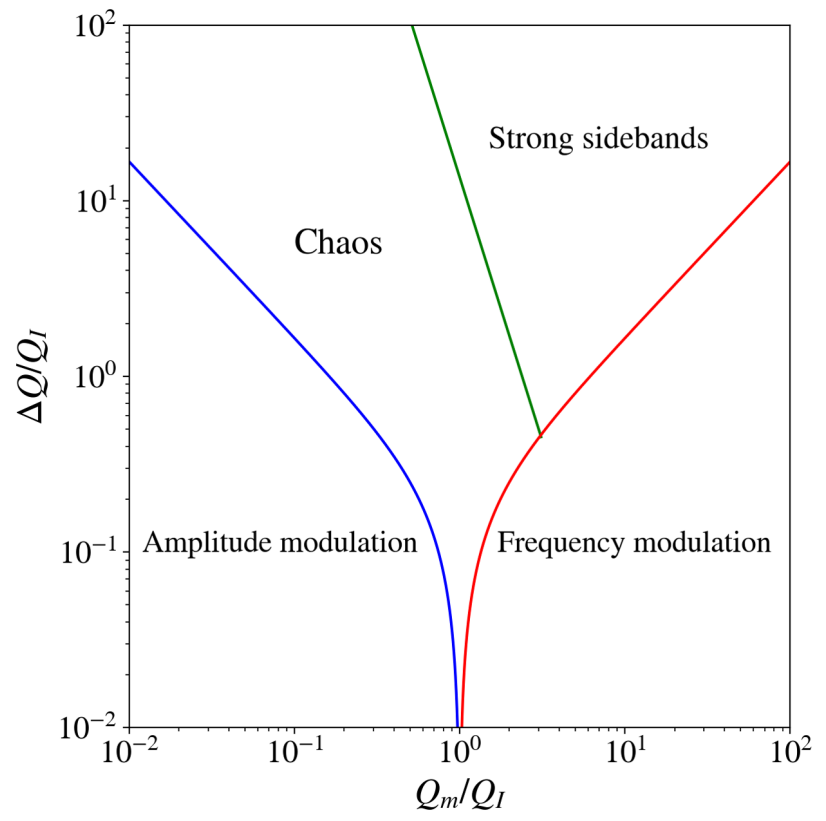

FIG. 1. The four regimes in the tune modulation parameter space for a modulation tune $Q_{m}$, a modulation depth $\Delta Q$, an island tune $Q_{I}$ [Eq. (9)] and the sixth-order resonance $(N=6)$ [17,32-34]. The blue, red, and green limits are computed from Eq. (10a), (10b), (10c), respectively.

affecting their stability (amplitude modulation regime). Second, increasing the frequency leads to the appearance of sideband islands around the resonance, spaced by the modulation tune at $k \cdot Q+m \cdot Q_{m}=n$ with $k, m, n$ integers. For small modulation depths, the islands occupy a limited portion of the phase space (frequency modulation). As the modulation depth increases, high-order sideband islands become significant (strong sidebands). The combination of a strong modulation in terms of amplitude and a frequency below a critical value, results in the overlap of the resonance islands, hence leading to chaotic motion (chaos). The limits of the four regimes are computed as [34]:

$$
\begin{aligned}
\left(\frac{\Delta Q}{Q_{I}}\right)\left(\frac{Q_{m}}{Q_{I}}\right) & =\frac{1}{6}, \\
\left(\frac{\Delta Q}{Q_{I}}\right)\left(\frac{Q_{m}}{Q_{I}}\right)^{-1} & =\frac{1}{6}, \\
\left(\frac{\Delta Q}{Q_{I}}\right)^{\frac{1}{4}}\left(\frac{Q_{m}}{Q_{I}}\right)^{\frac{3}{4}} & =\frac{4}{(6 \pi)^{\frac{1}{4}}} .
\end{aligned}
$$

The limits defined by Eq. (10a), (10b), (10c) are indicated with blue, red, and green, respectively, in Fig. 1.

Figure 1 shows that the transition to a chaotic state occurs when crossing the boundaries either from the amplitude modulation regime or through the excitation of sidebands (frequency modulation, strong sidebands regimes). 
The present paper investigates the effect of power supply ripple in a frequency range from $50 \mathrm{~Hz}$ to $10 \mathrm{kHz}$. Subsequently, the regimes of interest in the aforementioned parameter space are the frequency modulation, strong sidebands, and chaos, where emittance growth and particle losses occur through the excitation of sideband islands. For this reason, the present paper relies on the investigation of the sidebands and their impact on the beam performance, while the transition to chaos from the amplitude modulation regime is not discussed as it mainly refers to low-frequency tune modulation.

In two degrees of freedom and in the presence of a tune modulation $Q_{m}$, the resonance diagram is computed as:

$$
k \cdot Q_{x}+l \cdot Q_{y}+m \cdot Q_{m}=n,
$$

where $k, l, m, n$ are integers, $N=|k|+|l|$ is the resonance order and $m$ the sideband order. Figure 2 indicates the working point (black star-shaped marker) and illustrates Eq. (11) for a modulation at $100 \mathrm{~Hz}$ (left) and $800 \mathrm{~Hz}$ (right). The gray lines represent the nominal resonances in the absence of a tune modulation. The first sideband ( $m=1)$ of the linear coupling resonance $(N=2$, blue) and the third $(N=3$, cyan), fourth $(N=4$, green), fifth ( $N=5$, orange), and sixth $(N=6$, red) order resonance is also depicted. The solid and dashed lines illustrate the normal ( $l$ is an even number) and skew ( $l$ is an odd number) resonances, respectively.

The sidebands are always parallel to the main resonances and they are located at a distance that is proportional to the modulation tune, an effect that is clearly shown in Fig. 2 (left) with the $m=1$ and $N=2$ resonance (blue). As the modulation frequency increases, the sidebands, such as $m=1$ and $N=2$, are driven further away from the working point. At the same time, sidebands of excited resonances that are not in the vicinity of the working point reach the betatron tune spread, such as the sideband of the $N=3$ resonance (cyan) in Fig. 2 (right). Consequently, depending on the selected working point, the resonance strengths and the modulation frequency, the sidebands of lower or higher-order resonances might reach the betatron tune spread.

\section{BEAM-BEAM INTERACTION WITH POWER SUPPLY RIPPLE AND SYNCHROTRON OSCILLATIONS}

In the HL-LHC, luminosity leveling techniques are required to achieve a constant luminosity of $5 \times 10^{34} \mathrm{~cm}^{-2} \mathrm{~s}^{-}$ as envisaged in the nominal scenario $[2,36]$. The luminosity degradation resulting from the intensity reduction from $2.2 \times$ $10^{11}$ to $1.2 \times 10^{11}$ protons per bunch due to the proton-proton collisions, will be compensated by reducing the $\beta$-functions at the interaction points (IPs) of the high luminosity experiments (IP1 and 5) from $\beta^{*}=64 \mathrm{~cm}$ to the unprecedented value of $15 \mathrm{~cm}$ with the achromatic telescopic squeezing (ATS) scheme [37].

To reach an integrated luminosity of $250 \mathrm{fb}^{-1}$ per year, the HL-LHC will also operate with low beam emittances, high intensities and strong beam-beam interactions. In addition to the incoherent effects, the mitigation of collective instabilities requires the operation of the accelerator in a high chromatic and octupolar current regime. A chromaticity of $Q^{\prime}=15$ in both transverse planes is currently considered, controlled by the chromatic arc sextupoles, and strong Landau octupoles that result in amplitude detuning coefficients $\alpha_{n_{1} n_{2}}=\partial Q_{n_{1}} / \partial\left(2 J_{n_{2}}\right)$ with $n_{1}, n_{2} \in\{x, y\}$ equal to $\alpha_{x x}=-20.5 \times 10^{4} \mathrm{~m}^{-1}, \alpha_{x y}=6.8 \times 10^{4} \mathrm{~m}^{-1}$, $\alpha_{y y}=-20.6 \times 10^{4} \mathrm{~m}^{-1}$ as computed from the PTC-normal module of the MAD-X code [38]. As an alternated horizontal and vertical crossing scheme is used for the two high-luminosity experiments, the tune spread induced by
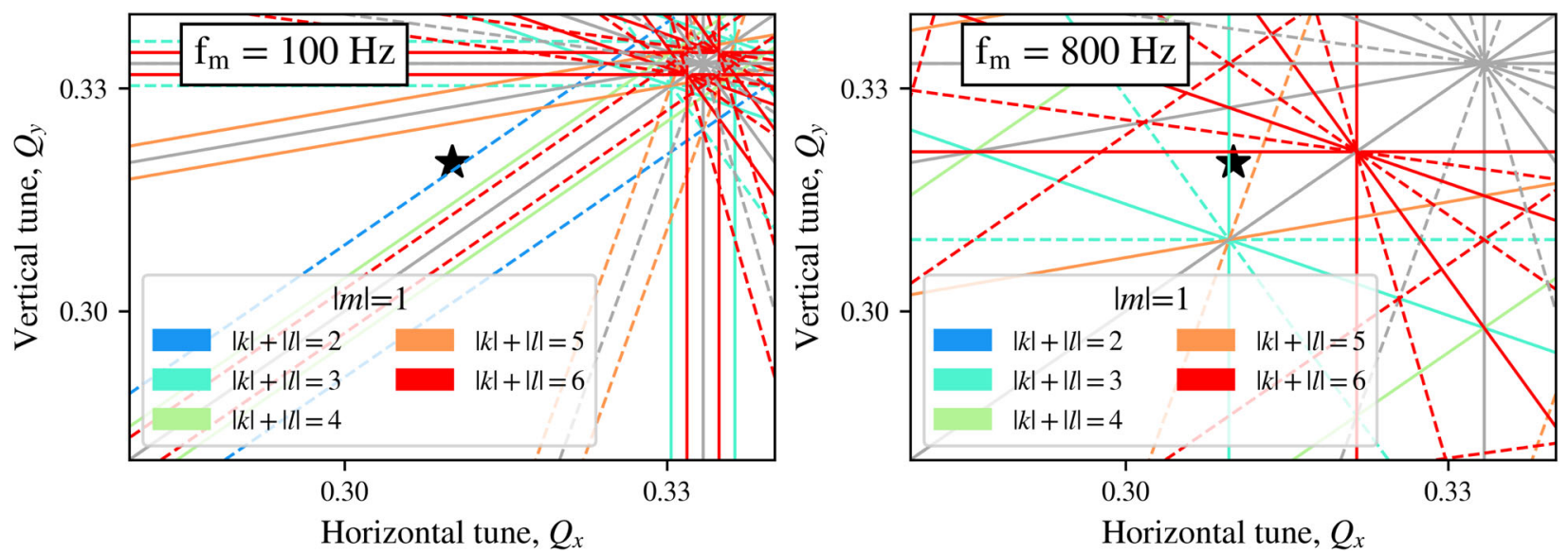

FIG. 2. An illustration of Eq. (11) for the nominal resonances (gray) and the first sideband $(m=1)$ of the linear coupling resonance $(N=|k|+|l|=2$, blue), the third $(N=3$, cyan), fourth $(N=4$, green $)$, fifth $(N=5$, orange), and sixth $(N=6$, red) order resonance for a tune modulation at $100 \mathrm{~Hz}$ (left) and $800 \mathrm{~Hz}$ (right). The working point is also illustrated (black). The solid and dashed lines illustrate the normal (even value of $l$ ) and skew (odd value of $l$ ) resonances, respectively. 
long-range beam-beam interactions is similar to the one induced by an octupole [39]. The amplitude detuning coefficients due to long-range beam-beam encounters are equal to $\alpha_{x x}=22 \times 10^{4} \mathrm{~m}^{-1}, \alpha_{x y}=-34.6 \times 10^{4} \mathrm{~m}^{-1}$, $\alpha_{y y}=24.9 \times 10^{4} \mathrm{~m}^{-1}$. This configuration results in significant nonlinearities.

Furthermore, a key component in the HL-LHC project is the upgrade of the interaction regions (IRs) around IP1 and 5. An important modification concerns the inner triplet layout, which provides the final focusing of the beam in the two high-luminosity experiments. Reducing the $\beta^{*}=$ $15 \mathrm{~cm}$ at the two IPs results in a maximum $\beta$-function of $21 \mathrm{~km}$ at the quadrupoles of the inner triplet, rendering the beam more sensitive to magnetic field fluctuations at this location. Due to the combined effect of the significant nonlinearities that arise from this configuration and the higher $\beta$-functions, a larger sensitivity to power supply ripple is anticipated.

The unprecedented high-values of the maximum $\beta$-functions at these locations, in combination with the next triplet generation based on niobium-tin technology that is currently being developed, motivates the need to perform a complete analysis on the beam performance implications of a modulation in the quadrupole gradients. Therefore, our investigation focuses on the four quadrupoles, namely $Q_{1}, Q_{3}$ (MQXFA magnets) and $Q_{2 \mathrm{a}}, Q_{2 \mathrm{~b}}$ (MQXFB magnets) and the three trim circuits, which are electrically powered in series. Simulations with power supply ripple in these circuits left and right of the two IPs are conducted for the simulated parameters shown in Table II. These parameters correspond to the end of the luminosity leveling during collisions in the nominal operational scenario [36].

The simulations are performed using the single-particle symplectic tracking code, SIXTRACK [40,41]. Only one beam is tracked around the element-by-element HL-LHC lattice, corresponding to Beam 1, and the weak-strong approximation is used for the beam-beam effects, as the charge distribution of the strong beam is not varied. Both head-on and long-range interactions are considered, as well as other important nonlinear fields such as the chromatic sextupoles and Landau octupoles. Studies with both the nominal and the optimized working point are performed, the values of which are presented in Table II. The optimized betatron tunes have been derived from DA studies without power supply ripple, which have shown that operating with such a working point can improve the DA and thus, the beam lifetime [42].

To simulate power supply ripple with a single tone resulting in a tune modulation described by Eq. (3), the normalized strengths of $Q_{1}, Q_{2 \mathrm{a}}, Q_{2 \mathrm{~b}}$, and $Q_{3}$ in the IRs left and right of IP1 and 5 are modulated with a sinusoidal function:

$$
\Delta k(t)=A_{r} \cos \left(2 \pi f_{m} t+\phi_{m}\right),
$$

TABLE II. The simulation parameters of the HL-LHC lattice at the end of the luminosity levelling during collisions based on the nominal operational scenario [36].

\begin{tabular}{lc}
\hline \hline Parameters (unit) & HL-LHC (values) \\
\hline Beam energy (TeV) & 7 \\
Bunch population (protons) & $1.2 \times 10^{11}$ \\
Bunch spacing (ns) & 25 \\
RMS bunch length $(\mathrm{cm})$ & 7.5 \\
RMS energy spread & $1.1 \times 10^{-4}$ \\
Beam-beam parameter $\left.\xi_{x, y}, Q_{y}\right)$ & $5.8 \times 10^{-3}$ \\
Nominal betatron tunes $\left(Q_{x}, Q_{x t}^{\text {opt }}, Q_{y}^{\text {opt }}\right)$ & $(0.31,0.32)$ \\
Optimized betatron tunes $(0.315,0.32)$ \\
Normalised emittance $(\mu \mathrm{m} \mathrm{rad})$ & 2.5 \\
Chromaticity $Q_{x, y}^{\prime}$ & 15 \\
Landau octupoles $(\mathrm{A})$ & -300 \\
IP1/5 half crossing angle $(\mu \mathrm{rad})$ & 250 \\
IP1/5 $\beta_{x, y}^{*}$ (cm) & 15 \\
Total rf voltage $(\mathrm{MV})$ & 16 \\
Synchrotron frequency $(\mathrm{Hz})$ & 23.8 \\
Revolution frequency $(\mathrm{kHz})$ & 11.245 \\
Harmonic number & 35640 \\
Bucket half height & $3.43 \times 10^{-4}$ \\
Relative momentum deviation $\delta p / p$ & $27 \times 10^{-5}$ \\
Maximum $\beta$-functions $\beta_{x}^{\max }, \beta_{y}^{\max }(\mathrm{km})$ & $21.58,21.12$ \\
\hline \hline
\end{tabular}

where $t$ is the time, $A_{r}$ the amplitude, $\phi_{m}$ the phase, $f_{m}=$ $Q_{m} \cdot f_{\text {rev }}$ the modulation frequency and $f_{\text {rev }}$ the revolution frequency listed in Table II. The amplitude of the function $A_{r}$ defines the maximum variation of the strength $\Delta k$ while the polarity of each magnet is preserved (e.g., focusing or defocusing in the horizontal plane). The absolute value of $A_{r}$ is assumed to be equal for all the quadrupoles, without however leading to the same modulation depth $\Delta Q$ due to variations of the $\beta$-functions. The horizontal and vertical $\beta$-functions of the inner triplet quadrupoles left and right of IP1 are presented in Table III, annotated with L1 and R1, respectively. These values are similar to the ones of IP5.

The phase of the injected ripple $\phi_{m}$ is considered equal to zero for all the circuits. This approximation does not correspond to a realistic scenario, as the switching clocks of the power supplies are not synchronized. Nevertheless, such a configuration is selected as it maximizes the overall impact of the power supply ripple. The modulation of the circuits accumulates when equal phases are considered, due to the anti-symmetric powering of the triplets left and right of each of the IPs. The relationship between the total modulation depth $\Delta Q$ induced by the four triplets and the amplitude of the excitation $A_{r}$ for $\phi_{m}=0$ can be approximated with $\Delta Q \approx 5480 . A_{r}$ as computed with MAD-X.

The quadrupoles in the inner triplet are powered by switch-mode power supplies [43]. In contrast to thyristor commutated technology, the voltage tones that are anticipated in the ripple spectrum are the switching frequencies of the power supplies and their harmonics, as well as a few low-order $50 \mathrm{~Hz}$ harmonics, namely 50, 150, 300, and 
TABLE III. The horizontal and vertical $\beta$-functions for the inner triplet quadrupoles left (L1) and right (R1) of IP1 at the end of the luminosity leveling (Table II).

\begin{tabular}{lrrcrr}
\hline \hline Quadrupole & $\beta_{x}(\mathrm{~km})$ & $\beta_{y}(\mathrm{~km})$ & Quadrupole & $\beta_{x}(\mathrm{~km})$ & $\beta_{y}(\mathrm{~km})$ \\
\hline$Q_{1}^{\mathrm{L} 1}$ & 5.76 & 4.56 & $Q_{1}^{\mathrm{R} 1}$ & 4.73 & 9.46 \\
$Q_{2 \mathrm{a}}^{\mathrm{L} 1}$ & 14.40 & 4.43 & $Q_{2 \mathrm{a}}^{\mathrm{R} 1}$ & 5.21 & 21.08 \\
$Q_{2 \mathrm{~b}}^{\mathrm{L}}$ & 21.64 & 5.85 & $Q_{2 \mathrm{~b}}^{\mathrm{R}}$ & 10.81 & 17.28 \\
$Q_{3}^{\mathrm{L} 1}$ & 9.34 & 20.50 & $Q_{3}^{\mathrm{R} 1}$ & 21.58 & 8.21 \\
\hline \hline
\end{tabular}

$600 \mathrm{~Hz}$ [44]. A summary of the switching frequencies $f_{\text {sw }}$ along with the inductances $L$ and the nominal currents $I_{\text {rated }}$ of the circuits under investigation are depicted in Table IV. Although it is anticipated that the switching frequencies and their harmonics will act as the main contributors to the ripple spectrum, such high frequencies are not expected to perturb the beam motion as they will be strongly attenuated by the shielding effect of the beam screen $[3,45]$.

As a realistic voltage spectrum is not presently available, the results of the simulations are compared to the power supply specifications, which are summarized as a function of the frequency in Table V. The specifications provide the maximum tolerated output voltage for an extended frequency bandwidth, without defining the actual amplitude and the voltage tones in the power supply ripple spectrum of the triplet. These values and the parameters of Table IV, are used to compute the maximum possible modulation depths of the four quadrupoles and the three trims left and right of the two IPs. Table VI presents these values for the horizontal plane and for two frequencies, $50 \mathrm{~Hz}$ and $600 \mathrm{~Hz}$.

In Eq. (1), the transfer function $T_{V \rightarrow I}$ is approximated by a simple RL circuit, $V(f)$ are the power supply specifications of Table $\mathrm{V}, T_{I \rightarrow B_{m}}=1$ and $T_{B_{m} \rightarrow B_{b}}=1$ as the beam screen attenuation is not included. This approach leads to

TABLE IV. The inductance $L$, the nominal current $I_{\text {rated }}$ and the switching frequency $f_{\mathrm{sw}}$ of the inner triplet circuits under consideration $[44,46]$.

\begin{tabular}{lccc}
\hline \hline Circuit & $L(\mathrm{mH})$ & $I_{\text {rated }}(\mathrm{kA})$ & $f_{\mathrm{sw}}(\mathrm{kHz})$ \\
\hline$Q_{1}, Q_{2 \mathrm{a}}, Q_{2 \mathrm{~b}}, Q_{3}$ & 255 & 18 & $20-200$ \\
Trim $Q_{1}$ & 69 & 2 & 50 \\
Trim $Q_{1 \mathrm{a}}$ & 34.5 & 0.06 & $45-200$ \\
Trim $Q_{3}$ & 69 & 2 & 50 \\
\hline \hline
\end{tabular}

TABLE V. The power supply specifications as a function of the frequency $[44,46]$.

\begin{tabular}{lccc}
\hline \hline Frequency & $V_{\text {rms }}(\mathrm{mV})$ & Frequency & $V_{\text {rms }}(\mathrm{mV})$ \\
\hline $50 \mathrm{~Hz}$ & 3.2 & $300 \mathrm{~Hz}-60 \mathrm{kHz}$ & 10 \\
$500 \mathrm{kHz}$ & 1 & $30 \mathrm{MHz}$ & 1 \\
\hline \hline
\end{tabular}

TABLE VI. The horizontal modulation depth of each circuit left (L1) and right (R1) of IP1 for $50 \mathrm{~Hz}$ and $600 \mathrm{~Hz}$ as computed from Eq. (1) and the parameters of Tables IV and V.

\begin{tabular}{lcc}
\hline \hline Circuit & $\Delta Q_{(50 \mathrm{~Hz})}\left(\times 10^{-7}\right)$ & $\Delta Q_{(600 \mathrm{~Hz})}\left(\times 10^{-7}\right)$ \\
\hline$Q_{1}^{L}, Q_{2 \mathrm{a}}^{L}, Q_{2 \mathrm{~b}}^{L}, Q_{3}^{L}$ & 1.79 & 0.47 \\
Trim $Q_{1}^{L}$ & 1.99 & 0.52 \\
Trim $Q_{1 \mathrm{a}}^{L}$ & 2.96 & 0.77 \\
Trim $Q_{3}^{L}$ & 3.31 & 0.86 \\
$Q_{1}^{R}, Q_{2 \mathrm{a}}^{R}, Q_{2 \mathrm{~b}}^{R}, Q_{3}^{R}$ & 1.39 & 0.36 \\
$\operatorname{Trim} Q_{1}^{R}$ & 1.48 & 0.39 \\
Trim $Q_{1 \mathrm{a}}^{R}$ & 2.95 & 0.77 \\
Trim $Q_{3}^{R}$ & 6.69 & 1.74 \\
\hline \hline
\end{tabular}

the overestimation of the maximum possible modulation depths from the power supply specifications as the lowpass filtering of the beam screen is not considered $[3,45]$.

\section{A. Power supply ripple spectrum with a single tone}

\section{Frequency map analysis with power supply ripple}

The existence and the impact of the sideband resonances described in Eq. (11) is validated with tracking simulations in the HL-LHC lattice in the presence of a single-tone power supply ripple in the inner triplets. The particles are tracked for $10^{4}$ turns at the nominal working point and with the simulation parameters of Table II. The initial conditions form a polar grid up to $6.1 \sigma$ in the configuration space, which in the present paper refers to the initial horizontal $x$ and vertical $y$ displacements of the particles, and with zero initial transverse momenta.

To disentangle the contribution from the power supply ripple and the synchro-betatron coupling, the synchrotron oscillations are not considered in this section. Nevertheless, to include the chromatic tune shift, the particles are placed off-momentum with a relative momentum deviation shown in Table II.

The turn-by-turn transverse displacements and momenta are retrieved and used for the frequency map analysis (FMA) [47-50]. In particular, the tune of each particle is computed for the first and last 3000 turns with the numerical analysis of fundamental frequencies (NAFF) algorithm [51-53]. The variation of the transverse tunes between the two time intervals $Q_{j_{1}}, Q_{j_{2}}$ with $j \in\{x, y\}$ defines the tune diffusion rate $D$ :

$$
D=\sqrt{\left(Q_{x_{1}}-Q_{x_{2}}\right)^{2}+\left(Q_{y_{1}}-Q_{y_{2}}\right)^{2}} .
$$

Figure 3 depicts the tune determination in the second time span color-coded with the logarithm of $D$ for four studies: in the absence of a tune modulation [Fig. 3(a)], which is used as a reference, and in the presence of a tune modulation with a modulation depth of $\Delta Q=5.5 \times 10^{-5}$ 

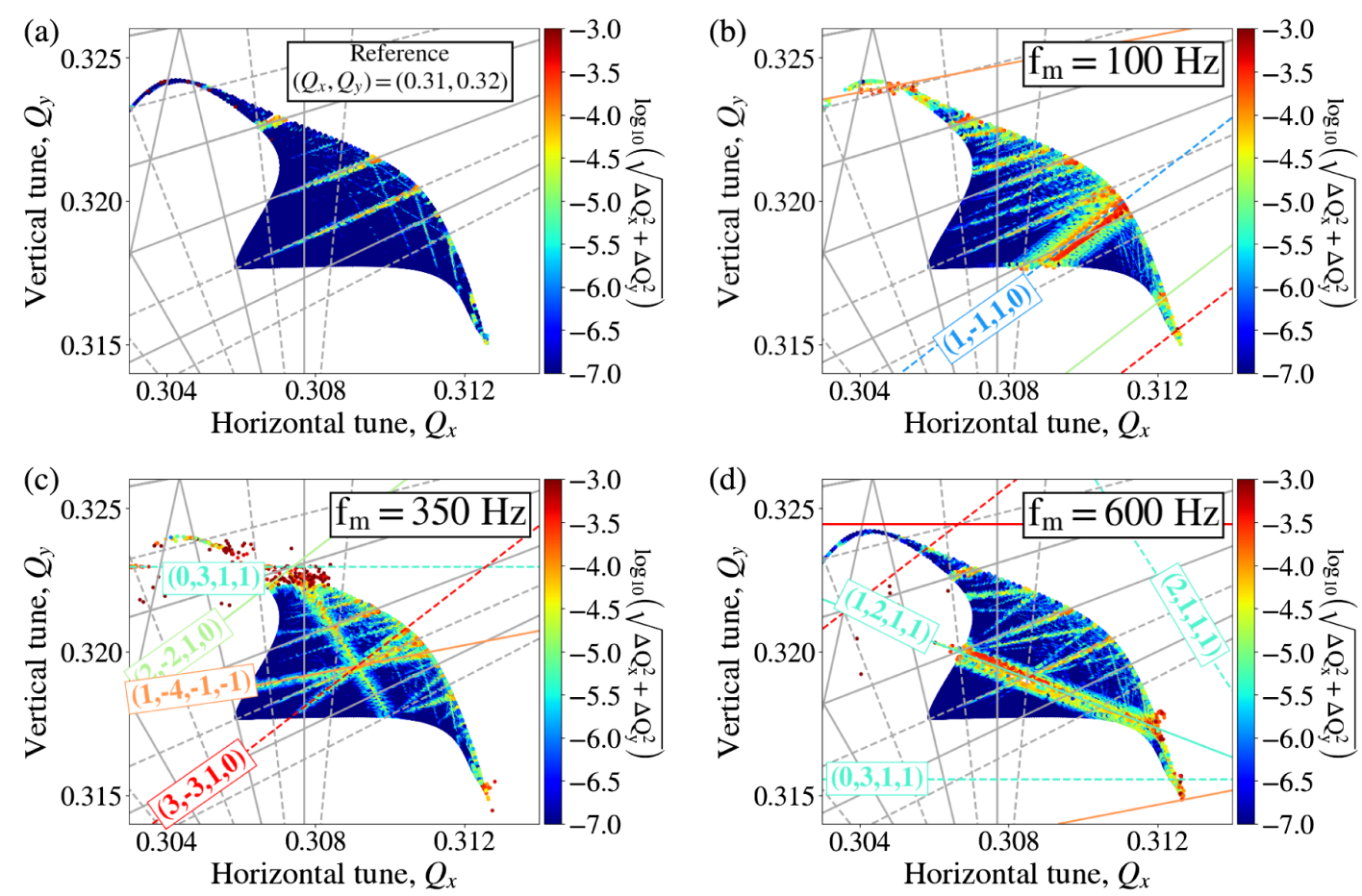

FIG. 3. Frequency map analysis without including synchrotron oscillations (a) in the nominal configuration and in the presence of a tune modulation with a modulation frequency of (b) $100 \mathrm{~Hz}$, (c) $350 \mathrm{~Hz}$, and (d) $600 \mathrm{~Hz}$ and a modulation depth of $\Delta Q=5.5 \times 10^{-5}$. A color code is assigned to the logarithm of the tune diffusion rate [Eq. (13)]. The nominal (gray) lines are illustrated along with the $m=1$ and $N=2$ (blue), $N=3$ (cyan), $N=4$ (green), $N=5$ (orange), and $N=6$ (red) resonances, annotated with the $(k, l, m, n)$ parameters of Eq. (11). The simulation parameters are listed in Table II.

at $100 \mathrm{~Hz}$ [Fig. 3(b)], $350 \mathrm{~Hz}$ [Fig. 3(c)], and $600 \mathrm{~Hz}$ [Fig. 3(d)]. The typical values of the tune diffusion rate $D$ extend from $10^{-7}$ (blue) to $10^{-3}$ (red). The gray lines represent the nominal resonances, while the colored lines illustrate the first sideband $(m=1)$ of the $N=2$ (blue), $N=3$ (cyan), $N=4$ (green), $N=5$ (orange), and $N=6$ (red) resonance. The $(k, l, m, n)$ parameters of Eq. (11) are also illustrated for each resonance with the same color code.

The review of the frequency maps shows that there is a good agreement between Eq. (11) and the tracking results. For a tune modulation at $100 \mathrm{~Hz}$ [Fig. 3(b)] the first sideband of the linear coupling resonance (blue) has a clear impact on the tune diffusion of the particles. A modulation at $350 \mathrm{~Hz}$ [Fig. 3(c)] leads to rapid losses due to the fact that the first sideband of the $N=3$ resonance (cyan) reaches high-amplitude particles and overlaps with the nominal resonances (gray lines), as well as with the $m=1$ and $N=4$ resonance (green). Finally, for a modulation at $600 \mathrm{~Hz}$ [Fig. 3(d)] the excitation of the $m=1$ and $N=3$ resonance (cyan) is visible. As this resonance affects a large portion of the phase space, a more critical impact is observed compared to the modulations at 50 or $100 \mathrm{~Hz}$. This explains why previous studies [22] report that the HL-LHC lattice is more sensitive to the higher frequency harmonics of 300 and $600 \mathrm{~Hz}$ than to the lower frequency harmonics anticipated at 50 and $150 \mathrm{~Hz}$.
Overall, in the presence of a tune modulation, the impact on the particles' tune diffusion rate depends on the resonance strengths, the position of the sidebands, the sideband and resonance order (lower orders have a more critical impact), the working point, and the actions of the affected particles. If the sidebands reach high amplitude particles rapid losses are observed in a limited amount of turns. The strong increase of the tune diffusion rate and eventually, of the beam losses depending on the proximity of a resonance has been shown in the past [54]. Tune modulation effects introduce a frequency-dependent diffusion mechanism, the impact of which significantly depends on the selected working point and the resonance strengths. Some modulation frequencies are more critical for the beam performance than others for the selected working point. Power supply ripple in the quadrupoles can affect the distribution even if the modulation frequency is not in the vicinity of the working point.

\section{Frequency map analysis with synchrotron oscillations}

The coupling of the synchrotron and the betatron motion in the presence of a nonvanishing chromaticity leads to a tune modulation with a modulation frequency equal to the synchrotron frequency. As the synchrotron frequency in the LHC (Table II) is lower than the ripple frequencies under consideration, the tracking is extended to $2 \times 10^{4}$ turns to 


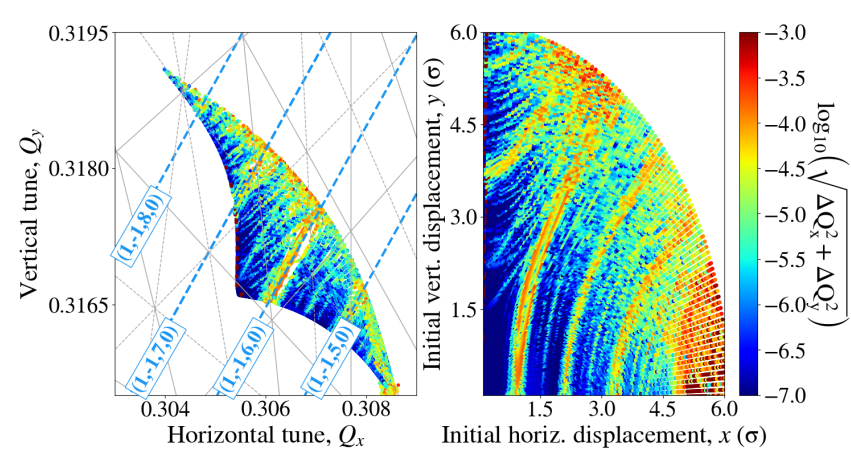

FIG. 4. Frequency map analysis (left) and initial configuration space (right) including synchrotron oscillations with chromaticity $Q^{\prime}=5$ in both transverse planes, relative momentum deviation $\delta p / p=27 \times 10^{-5}$, modulation depth $\Delta Q=1.35 \times 10^{-3}$ and modulation index $\beta_{m}=0.64$. The blue lines depict the sidebands $(m \leq 8)$ of the linear coupling resonance.

average over several modulation periods. The same distribution of particles is tracked including synchrotron oscillations in the nominal HL-LHC lattice without power supply ripple in the triplet.

The modulation index $\beta_{m}=1.9$, as computed from Eq. (4) and (5) with the parameters of Table II, exceeds the critical value of 1.41 [Eq. (8)]. Due to the appearance of strong sidebands, the NAFF algorithm returns the frequency determination of the sideband and not the one of the betatron tune. Therefore, to illustrate the frequency map including synchrotron oscillation the chromaticity is reduced to $Q^{\prime}=5$ in both transverse planes $\left(\beta_{m}=0.64\right)$.

Figure 4 demonstrates the FMA (left) and the initial configuration space (right) color-coded logarithm of $D$. The frequency map shows that high-order synchrotron sidebands $(m \leq 8)$ of the linear coupling resonance (blue) are excited. This underlines that, for the selected parameters, the chromatic tune modulation is in the strong sidebands regime of Fig. 1.

Although considering a constant relative momentum deviation $\delta p / p$ for all the particles in the distribution is essential to compute frequency maps, experimentally the initial longitudinal distribution extends over the whole bucket area. As the particles have different relative momentum deviations, the variation of the modulation index in the distribution leads to the reduction of the average modulation. The next section focuses on the investigation of the intensity evolution in a more realistic configuration.

\section{Intensity evolution simulations}

Intensity evolution studies require tracking with a realistic distribution of synchrotron oscillation amplitudes that spans the whole bucket height. Due to the excitation of additional resonances from the slow (synchrotron motion) and fast (power supply ripple) modulation, particles at the tails of the distribution diffuse and will eventually be lost. A detailed representation of the high amplitude particles is achieved by first, overpopulating the tails and then, assigning weights to the particles according to their initial amplitudes.

Specifically, the initial conditions form a 4D round distribution that extends up to $6 \sigma$ by sampling from a uniform distribution both in the initial configuration space and also in phase space. Longitudinally, the particles are uniformly placed in the bucket height extending up to its limit. The required number of particles for these simulations is derived from convergence studies (Appendix B). Overall, $9 \times 10^{4}$ particles are tracked for $10^{6}$ turns and the turn-by-turn transverse displacements and momenta are retrieved every $10^{3}$ turns.

Depending on the initial amplitudes of the particles in the transverse and longitudinal plane, a weight is assigned to each particle in the post-processing analysis. The weight defines the importance of each particle in the computations of the intensity. It is determined from the probability density function (PDF) of the simulated distribution. To simulate a Gaussian distribution in the horizontal and vertical plane, as well as in the longitudinal, the weight of a particle is computed as:

$$
w=\frac{\prod_{j=1}^{3} \frac{1}{\sqrt{2 \pi} \sigma_{j}} e^{\frac{-\left(x_{j}^{2}+p_{j}^{2}\right)}{2 \sigma_{j}}}}{\sum_{i} w_{i}},
$$

where $j$ iterates over the three planes, $x_{j}, p_{j}$ are the initial normalized position and momentum coordinates and the denominator denotes the normalization with the sum of the weights of all the particles. A mechanical aperture is defined in the post-processing at $5 \sigma$ to simulate the impact of the primary collimators. Particles reaching this limit are considered lost and the corresponding weights are set to zero.

Although Gaussian distributions are considered in the present paper, experimental observations show that the transverse bunch profiles in the LHC have overpopulated tails compared to the ones of a normal distribution $[55,56]$. The impact of non-Gaussian bunch profiles on the intensity evolution is treated in Appendix C.

Figure 5 depicts the intensity evolution as computed from the weighted distributions. First, the chromaticity and thus, the modulation depth is increased in steps [Fig. 5(a)] from 0 to 15 . The intensity evolution shows that the intensity degradation scales roughly linearly with the chromaticity increase. The instantaneous lifetime $\tau$ is computed from the intensity evolution $I$ as a function of time $t$ :

$$
I(t)=I_{0}(t) \cdot e^{-\frac{t}{\tau(t)}},
$$

where $I_{0}$ denotes the initial intensity. It is estimated using a sliding window in the intensity with a few thousand turns in each step. The fit is illustrated for a few cases in Fig. 5 

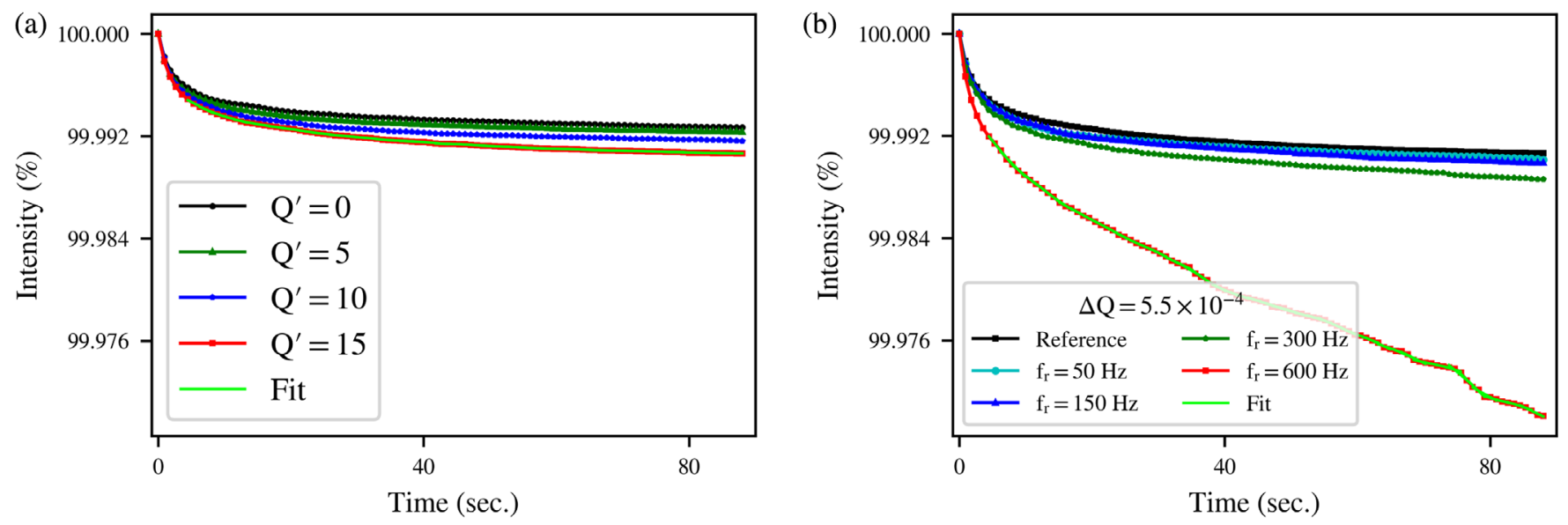

FIG. 5. (a) Intensity evolution in the HL-LHC lattice, including beam-beam interactions and with the simulation parameters of Table II, for a horizontal and vertical chromaticity of $Q^{\prime}=0$ (black), $Q^{\prime}=5$ (green), $Q^{\prime}=10$ (blue), and $Q^{\prime}=15$ (red). (b) Intensity evolution for $Q^{\prime}=15$ without power supply ripple (black) and in the presence of a tune modulation with $\Delta Q=5.5 \times 10^{-5}$ at $50 \mathrm{~Hz}$ (cyan), $150 \mathrm{~Hz}$ (blue), $300 \mathrm{~Hz}$ (green), and $600 \mathrm{~Hz}$ (red). An important impact is observed at $600 \mathrm{~Hz}$ due to the excitation of the $m=1$ and $N=3$ resonance. The fits for the estimation of the instantaneous lifetime [Eq. (15)] are also illustrated (light green).

(light green). The first few thousand turns are discarded from the computation of the lifetime as fast losses of particles close to the aperture limit occur. Increasing the chromaticity from 0 to 15 , the average instantaneous lifetime reduces from approximately $1600 \mathrm{~h}$ to $1100 \mathrm{~h}$. The fact that the instantaneous lifetime is not estimated with a simple single exponential fit but with a sliding window to obtain a more accurate representation of its evolution is illustrated in Fig. 15 of Appendix C.

For a constant chromaticity of $Q^{\prime}=15$ in both planes, the frequency of the tune modulation induced by power supply ripple is varied for a constant modulation depth $\Delta Q=5.5 \times 10^{-5}$ [Fig. 5(b)]. Although for a constant modulation depth the modulation index $\beta_{m}$ decreases with increasing frequency, the impact on the intensity is much more severe for $600 \mathrm{~Hz}$ (red) compared to 50 (cyan), 150 (blue), and $300 \mathrm{~Hz}$ (green). A reduction of the average lifetime from $\approx 1100 \mathrm{~h}$ to $\approx 750 \mathrm{~h}$ is computed by increasing the modulation frequency from $50 \mathrm{~Hz}$ to $300 \mathrm{~Hz}$ and to $\approx 130 \mathrm{~h}$ for $600 \mathrm{~Hz}$.

\section{Power supply ripple thresholds with parametric dynamic aperture scans}

Parametric simulations are performed to explore the impact of various modulation frequencies and depths on the DA and to compare the tracking results to the power supply specifications. The duration of the tracking is $10^{6}$ turns, which corresponds to $\approx 90$ seconds of beam collisions. For each study, a different combination of the modulation frequency and depth is selected in order to conduct a scan in the tune modulation parameter space. The frequency range spans over all $50 \mathrm{~Hz}$ harmonics up to $10 \mathrm{kHz}$. For each frequency, the amplitude of the excitation $A_{r}$ is increased and the total modulation depth $\Delta Q$ due to the contribution of all the circuits is computed. In these simulations, the chromatic tune modulation is also included with $\Delta Q=4 \times 10^{-3}$ as computed from the values of chromaticity and relative momentum deviation presented in Table II. The minimum DA across all the angles in the initial configuration space is computed.

Figure 6 presents the modulation frequency as a function of the modulation depth in a color plot of the minimum DA. The parametric scan defines the modulation depth threshold for each modulation frequency beyond which a significant DA reduction is foreseen (white to red). As an average,

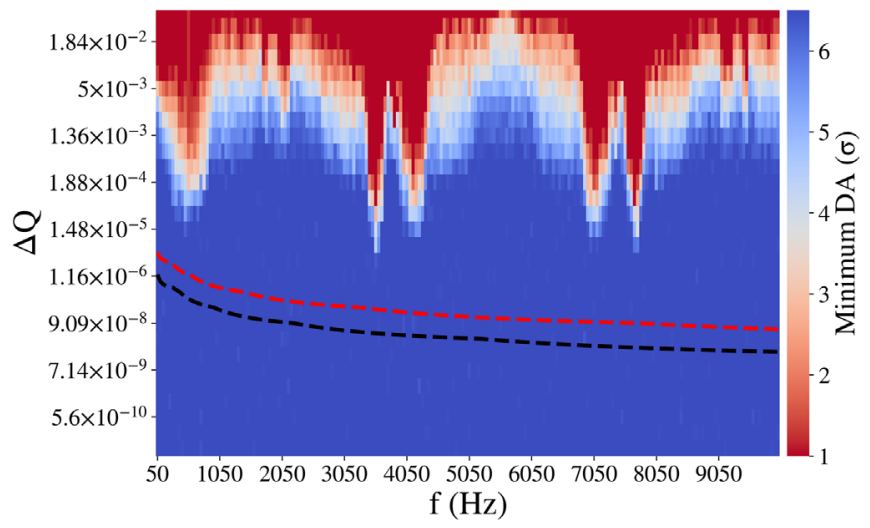

FIG. 6. Critical tune modulation depth $\Delta Q$ as a function of modulation frequency $f_{m}$ color-coded with the minimum DA in the presence of power supply ripple in the inner triplet quadrupoles. The simulation parameters are listed in Table II and the tracking is performed in the element-by-element HL-LHC lattice, including beam-beam interactions and chromatic tune modulation with $\Delta Q=4 \times 10^{-3}$. The red and black lines represent the sum and the root mean square of the contribution from all the circuits, respectively, computed from the power supply specifications of Table VI and Eq. (16). 
a maximum limit in the order of $\Delta Q=10^{-4}$ in the modulation depths can be defined; however the strong dependency on the modulation frequency is evident and, as expected from the frequency maps, there are regimes with increased sensitivity to power supply ripple.

Next, the maximum possible modulation depth is computed from the power supply specifications (Table VI). For each triplet, the contribution of the four main power supplies and the three trim converters are considered. The sum $\Delta Q_{\max }$ and the root mean square $\Delta Q_{\mathrm{rms}}$ modulation depths from all triplets is computed as:

$$
\begin{aligned}
\Delta Q_{\mathrm{max}} & =\sum_{a=1}^{4} \sum_{b=1}^{4} \Delta Q_{\mathrm{a}, \mathrm{b}}, \\
\Delta Q_{\mathrm{rms}} & =\sqrt{\sum_{a=1}^{4} \sum_{b=1}^{4} \Delta Q_{\mathrm{a}, \mathrm{b}}^{2},}
\end{aligned}
$$

where $a, b$ refer to the four triplets and the four circuits (Table V), respectively. The sum (red dashed) and the root mean square (black dashed) are illustrated in Fig. 6.

A comparison shows that the threshold modulation depths from simulations exceeds the maximum possible depths from the power supply specifications by approximately two orders of magnitude. Therefore, the much larger tolerances defined by the DA simulations suggest that, by considering individual tones, the ripple in the inner triplet, combined with large chromaticity values, will not pose a limitation for the beam performance.

\section{A review of the high-sensitivity power supply ripple regimes}

Based on the color plot of Fig. 6, a more detailed analysis is performed around the frequency regimes with a higher sensitivity to power supply ripple. Two ripple modulation depths, $\Delta Q=10^{-4}$ and $\Delta Q=10^{-3}$, illustrate the transition from slight to significant DA reduction.
The modulation frequency is $600 \mathrm{~Hz}$ in both cases and the optimized working point is selected (Table II).

Figure 7 depicts the FMA in the reference conditions (left) and with a tune modulation at $600 \mathrm{~Hz}$ for $\Delta Q=10^{-4}$ (middle), and $\Delta Q=10^{-3}$ (right). The first sideband of the $N=3$ (cyan), $N=5$ (orange), and $N=6$ (red) resonance is depicted. The analysis of the frequency maps demonstrates that the critical impact on the DA when increasing the modulation depth is observed due to, first, the increase of the sideband resonance strength and, second, the appearance of higher-order sidebands.

Second, a higher sensitivity is observed around the regime of the betatron frequency $\left(f_{x} \approx 3.48 \mathrm{kHz}\right)$ and its alias, i.e., the folding of the betatron frequency around the revolution frequency $\left(f_{\text {rev }}-f_{x} \approx 7.76 \mathrm{kHz}\right)$. This observation is attributed to the dipolar effect of the triplets through feed-down. In particular, as the particle trajectories are not aligned to the magnetic center of the quadrupoles, an orbit modulation is also observed.

This is demonstrated by tracking a single particle in the HL-LHC lattice under the influence of a tune modulation at $600 \mathrm{~Hz}$ and $3.4 \mathrm{kHz}$. From the turn-by-turn data the power spectrum is computed as demonstrated in Fig. 8. In the former case (top), apart from the sidebands (red) around the betatron tune (black), a dipolar excitation is also visible (purple). As the excitation frequency is not in the vicinity of the betatron tune spread, the dipolar effect has no impact on the tune diffusion. In the second case (bottom), the frequency of one of the sidebands $(\approx 6.89 \mathrm{kHz})$ exceeds the Nyquist frequency of the turnby-turn acquisitions $(\approx 5.62 \mathrm{kHz})$ and it is aliased into the spectrum $(\approx 4.36 \mathrm{kHz})$. The dipolar excitation (purple) approaches the betatron tune spread and eventually, has an impact on the betatron motion.

The dipolar effect of the triplet eventually leads to the excitation of additional resonances for modulation frequencies close to the tune. For the horizontal plane these first-order resonances are
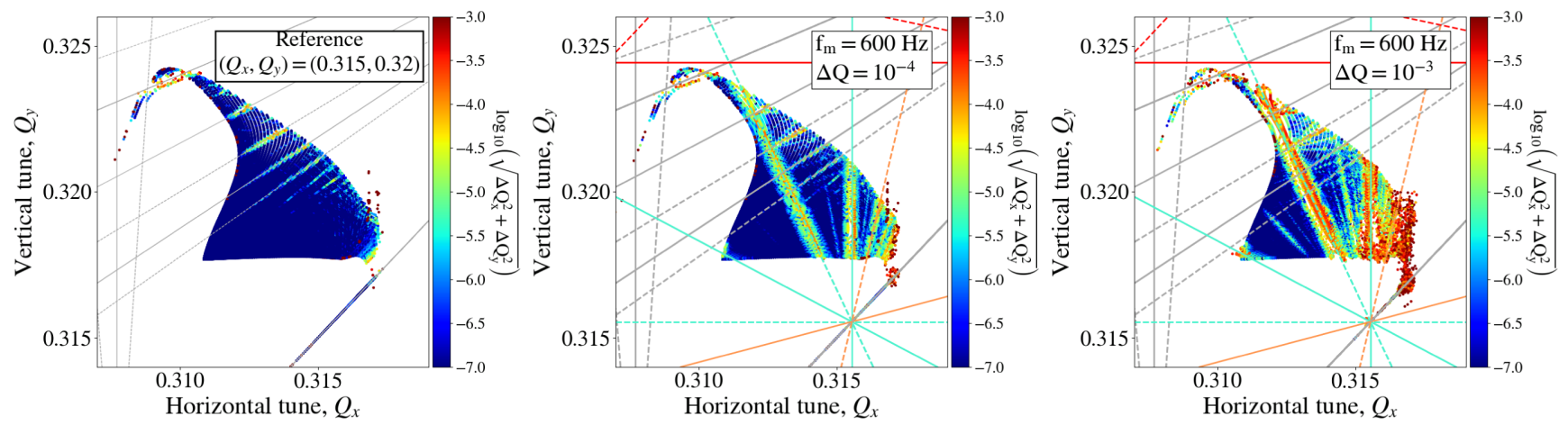

FIG. 7. The frequency maps at the optimized working point $\left(Q_{x}, Q_{y}\right)=(0.315,0.32)$, without considering synchrotron oscillations, with the reference conditions (left) and with a tune modulation at $600 \mathrm{~Hz}$ with $\Delta Q=10^{-4}$ (middle), and $\Delta Q=10^{-3}$ (right). The gray lines represent the nominal resonances, while the cyan lines the $m=1$ and $N=3$ resonance. 

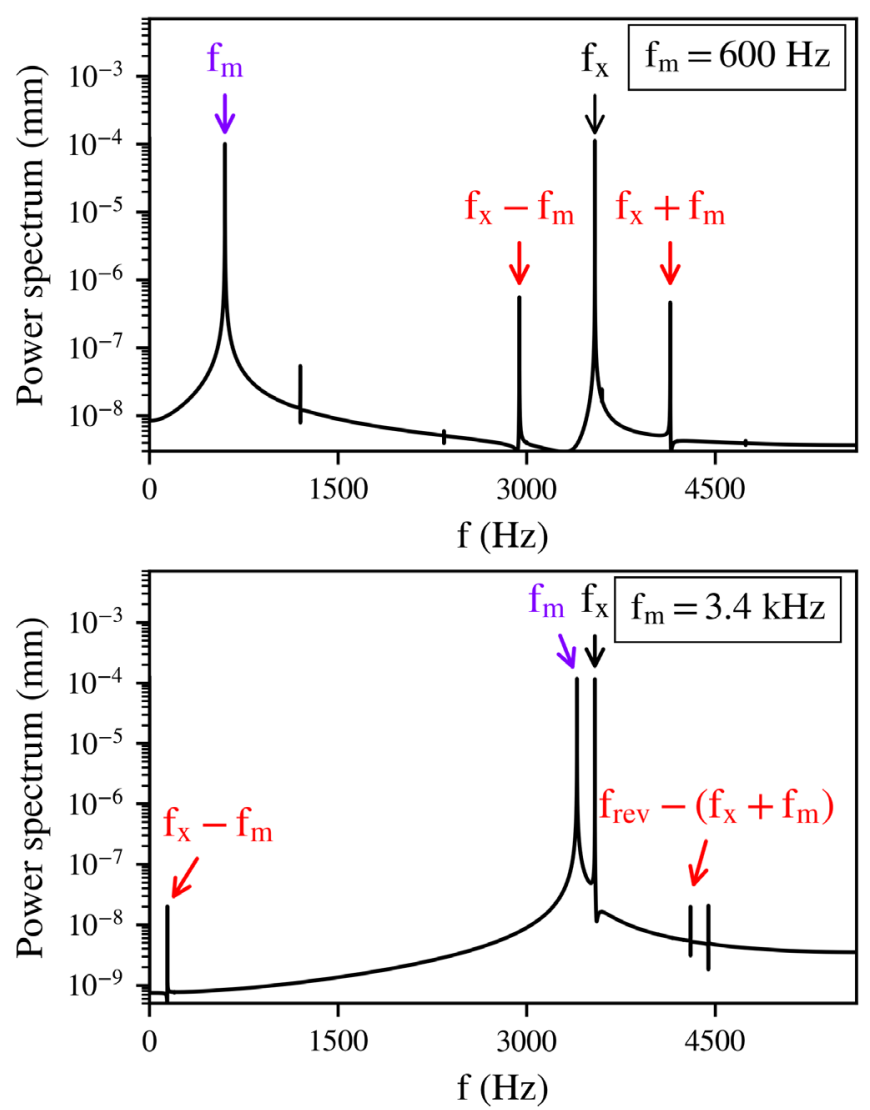

configuration space (right) for a tune modulation at a frequency in the vicinity of the working point $(3.55 \mathrm{kHz})$, which shows that the main contributor to the increase of the tune diffusion rate is the $m=1$ and $N=1$ resonance (purple).

\section{A visual tool to predict frequency sensitivity}

The parametric scan in the tune modulation parameter space presented in Fig. 6 is computationally challenging as it requires performing 6000 DA simulations. Moreover, the tracking must be repeated if the selected working point is modified. Although running the simulations up to $10^{6}$ turns is essential to determine the minimum modulation depth that leads to a DA reduction, it is not required to identify, in a fast way, the safest modulation frequencies for operation. A computationally simple and visual tool predicts the beam sensitivity to specific modulation frequencies based on the position of the sideband resonances. Only the knowledge of the betatron tune spread is needed for the unperturbed case, i.e., the reference conditions without power supply ripple.

The working principle of the method is presented in Fig. 10, which shows the betatron tune spread (left panel) and the initial configuration space (right panel) in the absence of power supply ripple (black). Based on the

FIG. 8. Power spectrum of a single particle, which is tracked in the HL-LHC lattice, in the presence of a tune modulation at $600 \mathrm{~Hz}$ (top) and $3.4 \mathrm{kHz}$ (bottom) that depicts the sidebands (red) around the betatron tune (black) and the dipolar excitation (purple) due to feed-down.

$$
Q_{x}=n \pm m \cdot Q_{m}
$$

The $N=1$ resonances appear as vertical lines in the tune diagram. Figure 9 illustrates the FMA (left) and the initial
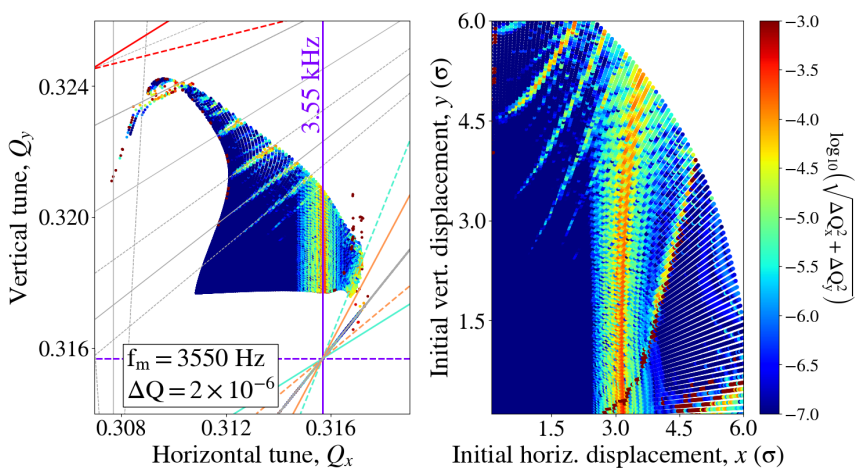

FIG. 9. Frequency map analysis (left) and initial configuration space (right) for the simulation parameters in Table II, a tune modulation at $3.55 \mathrm{kHz}$ and without considering synchrotron oscillations. The purple line represents the $m=1$ and $N=1$ resonance [Eq. (17)], which is the dipolar excitation at $3.55 \mathrm{kHz}$ in the horizontal plane.
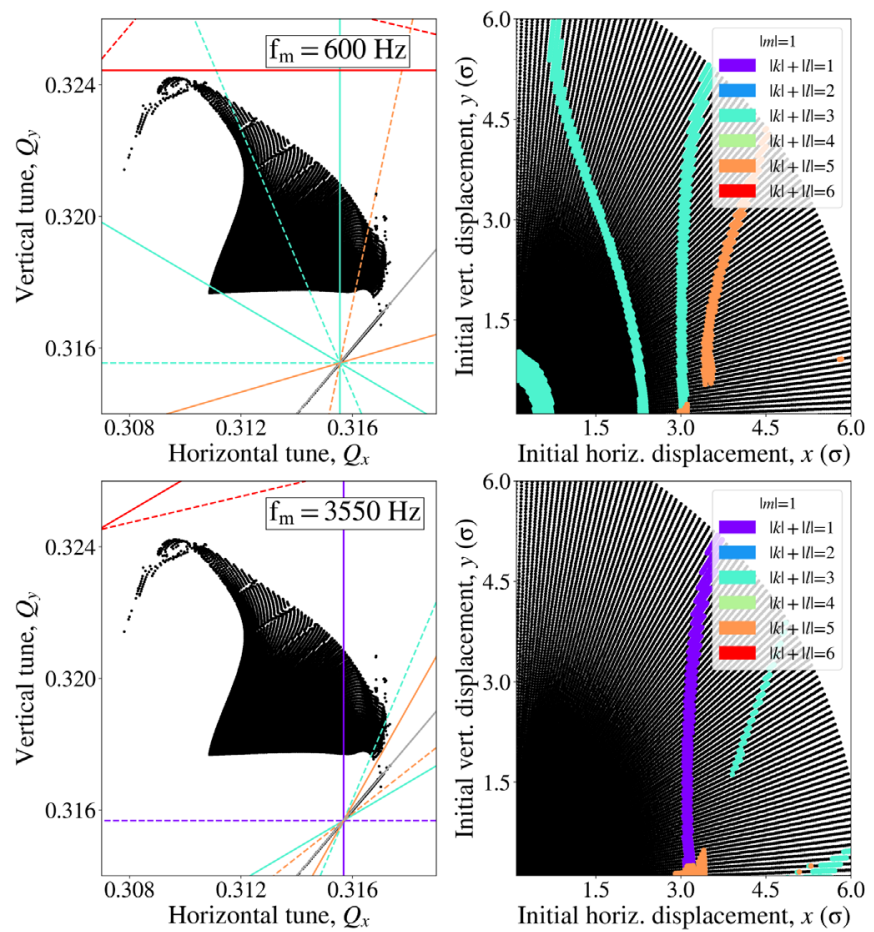

FIG. 10. The footprint (left panel) and the initial configuration space (right panel) for the simulation parameters of Table II and in the absence of power supply ripple (black). The first sideband ( $m=1$ ) of $N=1$ (purple), $N=2$ (blue) $N=3$ (cyan), $N=4$ (green), $N=5$ (orange) and $N=6$ (red) resonance is illustrated for a modulation at $600 \mathrm{~Hz}$ (top) and $3.55 \mathrm{kHz}$ (bottom) in both tune domain and configuration space. 
modulation tune, the $m=1$ and $N \leq 6$ resonances are computed from Eq. (11). Similarly to the previous frequency maps, a different color code is assigned to each resonance order.

Figure 10 depicts the sideband resonances in the vicinity of the footprint due to a modulation at $600 \mathrm{~Hz}$ (top) and $3.55 \mathrm{kHz}$ (bottom). For the selected working point, the $m=1$ and $N=3$ resonance (cyan) reaches the footprint for modulation at $600 \mathrm{~Hz}$.

Inspection of Fig. 10 (bottom) shows that the $m=1$ and $N=1$ resonance (purple), which is the dipolar excitation at $3.55 \mathrm{kHz}$, affects the footprint. As lower-order resonances have a more significant impact, a tune modulation at $3.55 \mathrm{kHz}(m=1$ and $N=1)$ is expected to be more detrimental to the beam performance than the modulation at $600 \mathrm{~Hz}(m=1$ and $N=3)$.

Then, depending on tune versus position, a resonance map is drawn connecting the tune space and the initial configuration space (Fig. 10 right panel). An example of the method is presented in the Appendix A. A modulation at $3.55 \mathrm{kHz}$ mainly affects the tails of the distribution, while a modulation frequency at $600 \mathrm{~Hz}$ has also an impact on the core. The representation of the resonances in the initial configuration space rather than in the tune domain allows for an easier correlation of the DA, which is computed in configuration space, with the tune diffusion.

A comparison of the predictions of Fig. 10 (bottom) to Fig. 9, which is computed with tracking simulations, shows that there is a good agreement between the two. In particular, the $m=1, N=1$ resonance has a strong impact on the particles with initial horizontal displacements at $3 \sigma$ and up to $5 \sigma$ in the vertical plane (Fig. 9 right panel), which is also predicted through the resonance mapping in Fig. 10 (bottom, right panel, purple). Therefore, using Eq. (11), determining whether the first sideband of the resonances up to a specific order reaches the footprint and mapping these sideband resonances in configuration space allows identifying the ones that affect the beam performance depending on the modulation frequency.

A similar analysis to Fig. 10 is performed for all the multiples of $50 \mathrm{~Hz}$ up to $10 \mathrm{kHz}$. Figure 11 presents the modulation frequency as a function of the resonance order $N$. For each modulation frequency, the previously described procedure is repeated to determine whether the $m=1$ or $m=2$ sideband up to the $N=6$ resonance is located in the vicinity of the working point. Then, a colorcode is assigned depending on whether the $m=1$ (red) or $m=2$ (orange) sideband affects the distribution. The frequency regimes with a red color, especially for low order resonances, are the modulation frequencies with the largest impact on the beam and should, therefore, be avoided. The orange regimes illustrate the $m=2$ sidebands and should be avoided only for large modulation depths. A blue color is assigned to the study if none of the resonances under consideration reach the footprint.

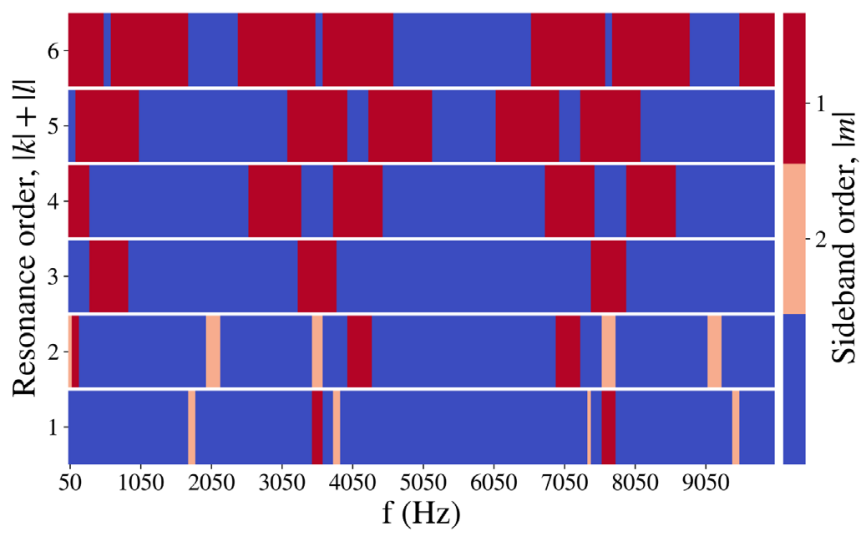

FIG. 11. The modulation frequency as a function of the resonance order $N=|k|+|l|$ as computed with the visual tool without tracking simulation and for the simulations parameters of Table II. A color code is assigned depending on whether the $m=1$ (red) or $m=2$ (orange) sideband reaches the footprint or not (blue) for each modulation frequency.

A comparison between the predictions (Fig. 11) and the results of the tracking simulations (Fig. 6) shows a good agreement between the two. The large impact on DA close to the betatron tune and its alias is due to the $m=1$ and $N=1$ resonance. In the vicinity of the betatron tune $(\approx 4.05 \mathrm{kHz})$ and its alias $(\approx 7.05 \mathrm{kHz})$, there is an additional high-sensitivity regime due to the $m=1$ and $N=2$ resonance. The impact on DA for modulation frequencies around $50-1050 \mathrm{~Hz}$ is attributed to the $m=1$ of the $N=2$ and $N=3$ resonances that reach the footprint for such modulation frequencies. For large modulation depths, a DA reduction is observed in the frequency region close to $(\approx 2.05 \mathrm{kHz})$, which is due to the $m=2, N=1$ and $N=2$ resonance. Finally, no impact on the DA was observed from the tracking simulations around the regime of $6.05 \mathrm{kHz}$, which is explained by the fact that no sideband up $N=6$ affects the footprint for such modulation frequencies.

In this way, the modulation frequencies with sideband resonances that do not reach the footprint can be easily identified and they can be distinguished in a fast way from the modulation frequencies with a critical impact. This visual tool can guide the selection of the power supply switching frequencies with the least possible impact on the beam dynamics in a fast way, for several working points and different optics.

\section{B. Including a power supply ripple spectrum with multiple tones}

The combined effect of the several voltage tones that are anticipated in the power supply ripple spectrum is estimated. The envisaged switching frequencies lie in a highfrequency regime (Table IV) and, as they are not expected to perturb the beam motion, they are not considered in the following analysis. In this context, the power supply ripple spectrum under consideration consists of the low-order 
TABLE VII. The maximum and root mean square modulation depths of the low order $50 \mathrm{~Hz}$ harmonics included in the simulations as computed from the power supply specifications (Table VI) and Eq. (16).

\begin{tabular}{lcc}
\hline \hline Frequency $(\mathrm{Hz})$ & $\Delta Q_{\mathrm{rms}}\left(\times 10^{-6}\right)$ & $\Delta Q_{\max }\left(\times 10^{-6}\right)$ \\
\hline 50 & 1.3 & 4.5 \\
150 & 0.8 & 2.8 \\
300 & 0.7 & 2.4 \\
600 & 0.3 & 1.2 \\
\hline \hline
\end{tabular}

$50 \mathrm{~Hz}$ harmonics. Table VII presents the root mean square $\Delta Q_{\text {rms }}$ and maximum $\Delta Q_{\max }$ modulation depth for each frequency as computed from Eq. (16). Three studies are performed: first, a baseline scenario in the absence of a tune modulation, second, including a ripple spectrum with multiple tones and $\Delta Q_{\mathrm{rms}}$ and finally, with $\Delta Q_{\max }$.

Figure 12 depicts the initial configuration space colorcoded with the tune diffusion rate in the absence of power supply ripple (left) and including the maximum modulation depths $\Delta Q_{\max }$ (right) at the frequencies listed in Table VII. The average tune diffusion rate, without considering synchrotron oscillations, increases from $\log _{10} D=-7.78$ in the baseline scenario to -7.43 when considering $\Delta Q_{\mathrm{rms}}$ and -7.18 with $\Delta Q_{\max }$. This small increase of the tune diffusion is mainly due to the tune modulation at $50 \mathrm{~Hz}$ and $600 \mathrm{~Hz}$.

Intensity evolution simulations, including synchrotron oscillations, are performed to estimate whether this slight increase in the tune diffusion rate can eventually enhance the particle losses. The results suggest that the considered ripple spectrum has an insignificant impact on the intensity compared to the reference case for both studies. It is concluded that the combined effect of multiple voltage tones in the ripple spectrum, with modulation depths that are extracted from the power supply specifications, will not affect the beam performance of the HL-LHC.

The tracking simulation studies presented in this paper include the most important nonlinear fields in the LHC

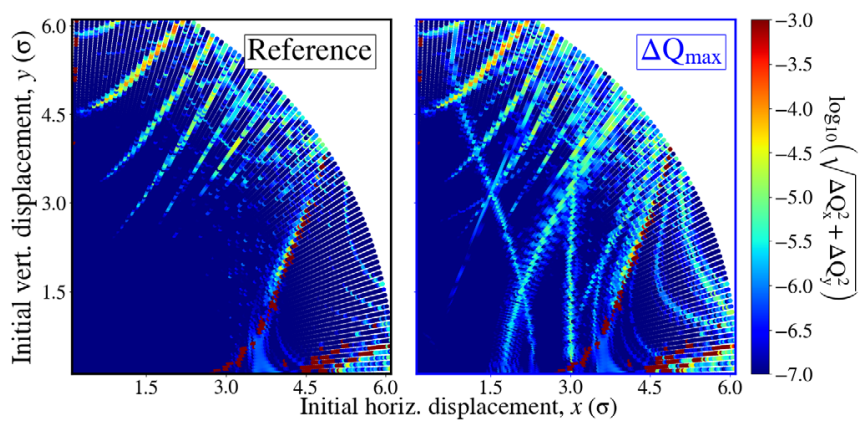

FIG. 12. The initial configuration space for the simulation parameters of Table II in the absence of power supply ripple (left) and including a tune modulation (right) with spectral components at $50,150,300$, and $600 \mathrm{~Hz}$ with the maximum modulation depths $\Delta Q_{\max }$ (Table VII). such as the long-range and head-on beam-beam, sextupoles and octupoles. Additional effects such as electron-cloud [57], which have been experimentally observed in the LHC, can potentially introduce additional nonlinearities and may enhance the impact of power supply ripple on the beam performance.

\section{CONCLUSIONS}

The increase of the $\beta$-functions in the HL-LHC inner triplets, resulting from the decrease of the beam size at the two IPs, and the presence of strong nonlinearities, combined with the new hardware that is currently being developed, motivates the need to perform a complete analysis on the repercussions of power supply ripple in future operation. These are based on simulations with parameters that correspond to the end of the luminosity leveling, a scenario that is the most critical in terms of power supply ripple. The tracking was performed in the element-by-element HL-LHC lattice, including head-on and long-range beam-beam interactions, arc sextupoles and octupoles. The modulation from the power supply ripple was combined with the chromatic tune modulation.

By individually scanning several modulation frequencies and depths the impact of the power supply ripple on the DA was computed. A comparison with the power supply specifications, without considering the shielding effects of the magnets that lead to a further attenuation of the ripple, showed that the maximum possible ripple level is approximately two orders of magnitude lower than the minimum modulation depths which reduce the DA.

The sensitivity of the beam performance to particular modulation frequencies stems from the excitation of sideband resonances that affect the particles' tune diffusion rate, a mechanism that strongly depends on the working point and the non-linear magnet strengths. This is the first time that FMAs have been used to demonstrate the excitation of sideband resonances, although their concept has been introduced in the past. Based on the position of the sideband resonances, a visual tool to determine in a fast way the safest modulation frequencies for operation such as the power supply switching frequencies in the inner triplet has been derived.

Including in the simulations the simultaneous effect of several voltage tones that are anticipated in the ripple spectrum, with modulation depths computed from the power supply specifications, it was concluded that the impact on the tune diffusion and eventually, on the lifetime is expected to be negligible.

The DA tolerances defined by the simulations must be experimentally verified in future operation through controlled quadrupolar excitations and experimental tune scans. Finally, to improve the validity of the simulations, a realistic power supply ripple spectrum must be included, in combination with a more accurate representation of the 
transfer function of the ripple spectrum from the voltage to the magnetic field.

A general analysis framework of simulation data has been developed. In particular, this paper demonstrated the importance of FMA in the investigation of power supply ripple, illustrated methods to define power supply ripple thresholds through DA scans and presented tools to determine the intensity evolution with weighted distributions. The methods can be applied to studies of several other types of power supply ripple and noise effects, as well as other present and future accelerators such as RHIC, EIC, and FCC.

\section{ACKNOWLEDGMENTS}

The authors gratefully acknowledge G. Arduini, R. De Maria, M. Fitterer, D. Gamba, R. T. Garcia and M. Martino for valuable suggestions and discussions on this work. Research supported by the HL-LHC project.

\section{APPENDIX A: RESONANCE MAP FROM TUNE DOMAIN TO INITIAL CONFIGURATION SPACE}

The tune of a particle with initial transverse displacements $\left(x_{0}, y_{0}\right)$ can be expressed in vector form as:

$$
\overrightarrow{v_{p}}=Q_{x} \overrightarrow{\nu_{x}}+Q_{y} \overrightarrow{\nu_{y}}
$$

where $Q_{x}$ and $Q_{y}$ denote the horizontal and vertical tune, respectively. Assuming two distinct points of the resonance line $\overrightarrow{v_{a}}=Q_{x_{a}} \overrightarrow{\nu_{x}}+Q_{y_{a}} \overrightarrow{\nu_{y}}$ and $\overrightarrow{r_{b}}=Q_{x_{b}} \overrightarrow{\nu_{x}}+Q_{y_{b}} \overrightarrow{\nu_{y}}$, the resonance line is expressed as:

$$
\overrightarrow{v_{r}}=\overrightarrow{v_{r_{b}}}-\overrightarrow{v_{r_{a}}} .
$$

In tune domain, the perpendicular distance of the particle's tune from the resonance line is

$$
d=\frac{\left|\left(\overrightarrow{v_{p}}-\overrightarrow{v_{r_{a}}}\right) \times \overrightarrow{v_{r}}\right|}{\left|\overrightarrow{v_{r}}\right|} .
$$

To determine whether the particle is affected by the resonance, a distance tolerance $\epsilon$ is defined:

$$
d<\epsilon .
$$

A good agreement between this method and tracking simulations has been found for $\epsilon=10^{-4}$. If the condition of Eq. (A4) is satisfied, the initial transverse positions of the particle are retrieved and the procedure is repeated for all the particles in the distribution. The method is then iterated for all the sideband resonances that reach the footprint.

For instance, in the presence of a tune modulation at $f_{m}=600 \mathrm{~Hz}$, a sideband resonance that reaches the footprint is $2 Q_{x}+Q_{y}+Q_{m}=1$. In this case, $\overrightarrow{v_{a}}=$ $0.31632 \overrightarrow{\nu_{x}}+0.314 \overrightarrow{\nu_{y}}$ and $\overrightarrow{v_{r_{b}}}=0.31032 \overrightarrow{\nu_{x}}+0.326 \overrightarrow{\nu_{y}}$.
Considering a particle with initial horizontal and vertical displacements $\left(x_{0}, y_{0}\right)=\left(0.93 \sigma_{x}, 4.52 \sigma_{y}\right)$ with $\overrightarrow{v_{p}}=$ $0.31266 \overrightarrow{\nu_{x}}+0.32120 \vec{\nu}_{y}$, Eq. (A3) yields $d=0.5 \times 10^{-4}$. As Eq. (A4) is satisfied, the particle is affected by the resonance and its initial conditions are used for the mapping. Iterating over all particles results in the projection of the resonance from the tune domain to the initial configuration space.

\section{APPENDIX B: CONVERGENCE STUDIES FOR INTENSITY EVOLUTION SIMULATIONS}

Convergence studies are performed to determine the suitable number of particles in the tracking simulations with weighted distributions that offers sufficient statistical significance for the intensity evolution estimations. Figure 13 depicts the intensity evolution for an increasing number of particles starting from $20 \times 10^{3}$ (blue) and extending up to $250 \times 10^{3}$ particles (purple). Based on the convergence tests, a minimum sample size of $80 \times 10^{3}$ (green) particles is sufficient to converge to an accurate intensity evolution estimation.

\section{APPENDIX C: NON-GAUSSIAN BUNCH PROFILES}

Studies of the LHC bunch profiles have shown that their tails are overpopulated in the transverse plane and underpopulated in the longitudinal plane compared to the ones of a Gaussian distribution [55]. To include non-Gaussian bunch profiles in the intensity evolution simulations, the PDF of a generalized Gaussian distribution is employed, the q-Gaussian distribution [56]. The parameter $q$ of the q-Gaussian distribution represents the weight of the tails. The normal distribution is retrieved as $q \rightarrow 1$, while $q>1$ describes a distribution with overpopulated or "heavier" tails compared to ones of the normal Gaussian distribution.

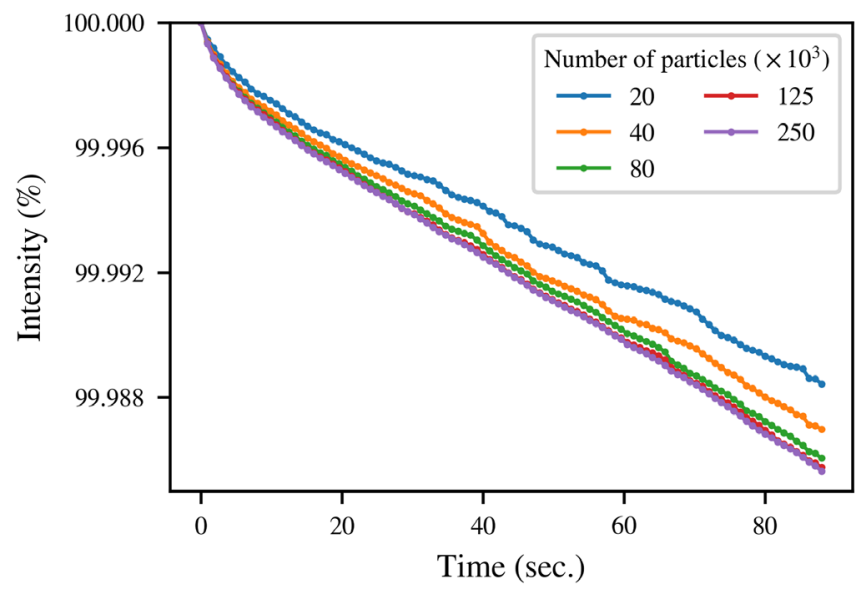

FIG. 13. Intensity evolution computed with $20 \times 10^{3}$ (blue), $40 \times 10^{3}$ (orange), $80 \times 10^{3}$ (green), $125 \times 10^{3}$ (red), and $250 \times 10^{3}$ (purple) particles and the weights from Eq. (14). 


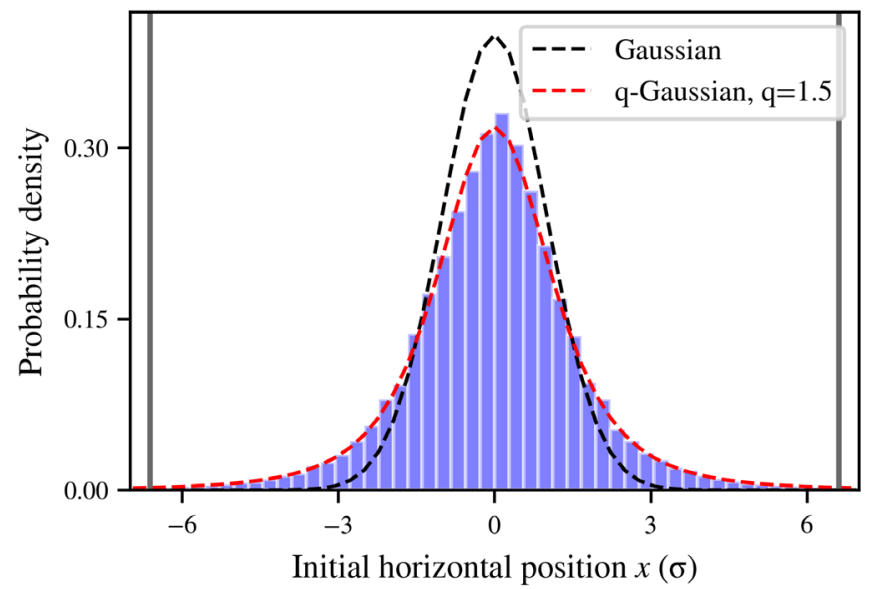

FIG. 14. Probability density of the horizontal initial positions normalized to the beam size (blue). The weights of Eq. (14) are computed with the q-Gaussian PDF and $q=1.5$ (red dashed line). The PDF of the normal distribution is also shown (black dashed line). The gray vertical lines illustrate the position of the primary collimators during the LHC physics operation in 2018.

Figure 14 depicts the probability density of the horizontal initial positions (blue) normalized with the beam size. The weights of Eq. (14) are computed with the q-Gaussian PDF and $q=1.5$ for both transverse planes, while a normal distribution is used for the longitudinal plane. The PDF of the normal ( $q=1$, black dashed line) and the q-Gaussian distribution $(q=1.5$, red dashed line $)$ are also illustrated. The comparison between the two clearly shows the overpopulation of the tails in the $q=$ 1.5 case. The gray vertical lines represent the physical position of the primary collimators during the LHC physics operation in $2018\left(6.7 \sigma, \epsilon_{n}=2 \mu \mathrm{m} \mathrm{rad}\right)$.

Bunch profiles like the one of Fig. 14 are used as initial conditions for the intensity evolution simulations. To study the impact of tail population on the beam lifetime, a series

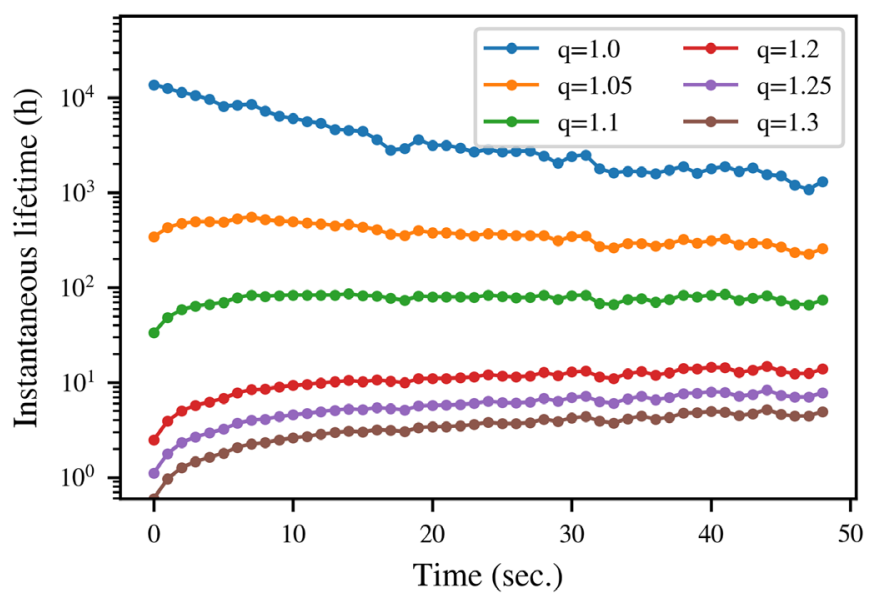

FIG. 15. Instantaneous lifetime computed with q-Gaussian distributions and $q=1$ (blue), $q=1.05$ (orange), $q=1.1$ (green), $q=1.2$ (red), $q=1.25$ (purple), and $q=1.3$ (brown). of studies are performed for different $q$-parameters in the transverse plane. The instantaneous beam lifetime is estimated by applying the fit of Eq. (15) with a sliding window in each case. Figure 15 presents the evolution of the instantaneous lifetime as a function of time for a $q$-parameter that spans from 1 (blue) to 1.3 (brown). The instantaneous lifetime strongly depends on the tail overpopulation. In particular, a reduction of the average instantaneous lifetime from $3200 \mathrm{~h}(q=1)$ to $3.7 \mathrm{~h}$ $(q=1.3)$ is observed. This observation highlights the importance of including realistic bunch profiles in the simulations for an accurate estimation of the beam lifetime.

[1] O. S. Brüning, P. Collier, P. Lebrun, S. Myers, R. Ostojic, J. Poole, and P. Proudlock, LHC Design Report, CERN Yellow Reports: Monographs (CERN, Geneva, 2004).

[2] G. Apollinari, I. B. Alonso, O. Brüning, P. Fessia, M. Lamont, L. Rossi, and L. Tavian, High-Luminosity Large Hadron Collider (HL-LHC), CERN Yellow Rep. Monogr. 4, 1 (2017).

[3] M. Morrone, M. Martino, R. De Maria, M. Fitterer, and C. Garion, Magnetic frequency response of High-Luminosity Large Hadron Collider beam screens, Phys. Rev. Accel. Beams 22, 013501 (2019).

[4] S. G. Peggs and R. Talman, Nonlinear problems in accelerator physics, Annu. Rev. Nucl. Part. Sci. 36, 287 (1986).

[5] A. Chao, D. Johnson, S. Peggs, J. Peterson, C. Saltmarsh, L. Schachinger, R. Meller, R. Siemann, R. Talman, P. Morton et al., Experimental Investigation of Nonlinear Dynamics in the Fermilab Tevatron, Phys. Rev. Lett. 61, 2752 (1988).

[6] T. Satogata, T. Chen, B. Cole, D. Finley, A. Gerasimov, G. Goderre, M. Harrison, R. Johnson, I. Kourbanis, C. Manz, N. Merminga, L. Michelotti, S. Peggs, F. Pilat, S. Pruss, C. Saltmarsh, S. Saritepe, R. Talman, C. G. Trahern, and G. Tsironis, Driven Response of a Trapped Particle Beam, Phys. Rev. Lett. 68, 1838 (1992).

[7] S. G. Peggs, Hamiltonian theory of the E778 nonlinear dynamics experiment, in 2nd ICFA Advanced Beam Dynamics Workshop (1988), p. 15, http://cds.cern.ch/ record/189293/files/131.pdf.

[8] X. Altuna, C. Arimatea, R. Bailey, T. Bohl, D. Brandt, K. Cornelis, C. Depas, F. Galluccio, J. Gareyte, R. Giachino, M. Giovannozzi, Z. Guo, W. Herr, A. Hilaire, T. Lundberg, J. Miles, L. Normann, T. Risselada, W. Scandale, F. Schmidt, A. Spinks, and M. Venturini, The 1991 dynamic aperture experiment at the CERN SPS, Tech. Rep. Nos. CERN-SL-91-43-AP, LHC-NOTE-171, CERN-LHC-Note-171 (CERN, Geneva, 1991).

[9] W. Fischer and M. Giovannozzi, Dynamic aperture experiment at a synchrotron, Phys. Rev. E 55, 3507 (1997).

[10] O. S. Brüning, Diffusion in A FODO cell due to modulation effects in the presence of nonlinear fields, Part. Accel. 41, 133 (1992).

[11] W. Fischer and T. Satogata, A simulation study on tune modulation effects in RHIC, Tech. Rep. (Brookhaven National Lab. (BNL), Upton, NY (United States), 1996). 
[12] G. Parzen, Tune modulation due to synchrotron oscillations and chromaticity, and the dynamic aperture, in Proceedings, 16th Particle Accelerator Conference and International Conference on High-Energy Accelerators, HEACC 1995: Dallas, USA, May 1-5, 1995 (1994) pp. 2798-2800, https://accelconf.web.cern.ch/p95/ ARTICLES/FAB/FAB04.pdf.

[13] G. Parzen, Tune modulation due to gradient ripple and the dynamic aperture, Tech. Rep. (Brookhaven National Lab., Upton, NY (United States), 1994).

[14] O.S. Brüning, M. Seidel, K. Mess, and F. Willeke, Measuring the effect of an external tune modulation on the particle diffusion in the proton storage ring of HERA, Tech. Report No. P00021147, 1994.

[15] O.S. Brüning and F. Willeke, Diffusion-like processes in proton storage rings due to the combined effect of nonlinear fields and modulation effects with more than one frequency, in Proceedings of the 4th European Particle Accelerator Conference, London, England, 1994 (EPSAG, London, UK, 1994).

[16] O. S. Brüning and F. Willeke, Reduction of particle losses in HERA by generating an additional harmonic tune modulation, in Proceedings of the Particle Accelerator Conference, Dallas, TX, 1995 (IEEE, New York, 1995), pp. 420-422, Vol. 1.

[17] F. Zimmermann, Emittance growth and proton beam lifetime in HERA, Ph.D. thesis, Hamburg U., 1993.

[18] O. S. Brüning, An analysis of the long-term stability of the particle dynamics in hadron storage rings, Ph.D. thesis, Hamburg U., 1994.

[19] S. Kostoglou, G. Arduini, L. Intelisano, Y. Papaphilippou, and $\mathrm{G}$. Sterbini, Impact of the $50 \mathrm{~Hz}$ harmonics on the beam evolution of the Large Hadron Collider, arXiv:2003 .00140 .

[20] W. Fischer, An experimental study on the long-term stability of particle motion in hadron storage rings, Ph.D. thesis, Hamburg U., 1995.

[21] Y. Papaphilippou and F. Zimmermann, Weak-strong beambeam simulations for the large hadron collider, Phys. Rev. Accel. Beams 2, 104001 (1999).

[22] M. Fitterer, R. De Maria, S. Fartoukh, and M. Giovannozzi, Beam dynamics requirements for the powering scheme of the HL-LHC triplet, in Proceedings, 6th International Particle Accelerator Conference (IPAC 2015): Richmond, Virginia, USA, May 3-8, 2015 (JACoW, Geneva, 2015), p. TUPTY035.

[23] B. V. Chirikov, Resonance processes in magnetic traps, Sov. J. At. Energy 6, 464 (1960).

[24] B. V. Chirikov, Research concerning the theory of non-linear resonance and stochasticity, Tech. Report No. CM-P00100691, 1971.

[25] B. V. Chirikov, A universal instability of many-dimensional oscillator systems, Phys. Rep. 52, 263 (1979).

[26] B. V. Chirikov, M. Lieberman, D. Shepelyansky, and F. Vivaldi, A theory of modulational diffusion, Physica D (Amsterdam) 14, 289 (1985).

[27] The Electron-Ion Collider (EIC), https://www.bnl.gov/eic/ (2020), accessed: 2020-05-26.

[28] The Future Circular Collider (FCC), https://fcc.web.cern .ch/ (2020), accessed: 2020-05-26.
[29] T. Suzuki, Synchro-betatron resonances, Part. Accel. 27, 157 (1989).

[30] H. Roder, Amplitude, phase, and frequency modulation, Proceedings of the Institute of Radio Engineers 19, 2145 (1931).

[31] A. W. Chao and M. Month, Particle trapping during passage through a high-order nonlinear resonance, Nucl. Instrum. Methods 121, 129 (1974).

[32] T. J. Satogata, Nonlinear resonance islands and modulational effects in a proton synchrotron, Ph.D. thesis, Northwestern U. (1993).

[33] T. Satogata and S. Peggs, Is beta modulation more or less potent than tune modulation?, in Conference Record of the 1991 IEEE Particle Accelerator Conference: Accelerator Science and Technology, May 6-9, 1991, San Francisco, California (IEEE, New York, 1991), p. 476.

[34] S. Peggs and T. Satogata, Introduction to Accelerator Dynamics (Cambridge University Press, Cambridge, England, 2017).

[35] S. Y. Lee et al., Experimental Determination of a Nonlinear Hamiltonian in a Synchrotron, Phys. Rev. Lett. 67, 3768 (1991).

[36] E. Metral et al., Update of the HL-LHC operational scenarios for proton operation, CERN Technical Report, 2018.

[37] S. Fartoukh, Achromatic telescopic squeezing scheme and application to the LHC and its luminosity upgrade, Phys. Rev. Accel. Beams 16, 111002 (2013).

[38] MAD-X, http://mad.web.cern.ch/mad/ (2019), accessed: 2019-12-13.

[39] S. Fartoukh, A. Valishev, Y. Papaphilippou, and D. Shatilov, Compensation of the long-range beam-beam interactions as a path towards new configurations for the high luminosity LHC, Phys. Rev. Accel. Beams 18, 121001 (2015).

[40] SixTrack, http://sixtrack.web.cern.ch/SixTrack/ (2019), accessed: 2019-11-26.

[41] R. D. Maria, J. Andersson, V. K. B. Olsen, L. Field, M. Giovannozzi, P. D. Hermes, N. Høimyr, S. Kostoglou, G. Iadarola, E. Mcintosh, A. Mereghetti, J. W. Molson, D. Pellegrini, T. Persson, M. Schwinzerl, E. H. Maclean, K. Sjobak, I. Zacharov, and S. Singh, SixTrack project: Status, runtime environment, and new developments, in Proceedings, 13th International Computational Accelerator Physics Conference, ICAP2018: Key West, FL, USA, 20-24 October 2018 (2019) p. TUPAF02, http://accelconf.web .cern.ch/icap2018/papers/tupaf02.pdf.

[42] N. Karastathis, R. De Maria, S. Fartoukh, Y. Papaphilippou, and D. Pellegrini, Refining the HL-LHC operational settings with inputs from dynamic aperture simulations: A progress report, J. Phys. Conf. Ser. 1067, 022005 (2018).

[43] E. Coulinge, J.-P. Burnet, and D. Dujic, High-current lowvoltage power supplies for superconducting magnets, in 2017 International Symposium on Power Electronics (Ee) (IEEE, New York, 2017), pp. 1-6.

[44] D. Gamba, G. Arduini, M. Cerqueira Bastos, J. M. C. De Portugal Martinez Vazquez, R. De Maria, M. Giovannozzi, M. Martino, and R. T. Garcia, Beam dynamics requirements for HL-LHC electrical circuits, CERN Technical Report No. CERN-ACC-2017-0101, 2017. 
[45] R. De Maria, T. Kroyer, and V. Shiltsev, Field fluctuation and beam screen vibration measurements in the LHC magnets, CERN Technical Report Nos. LHC-PROJECTReport-1041, CERN-LHC-PROJECT-Report-1041, 2007; revised version submitted on 2007-09-24 16:35:23.

[46] D. Gamba, G. Arduini, M. C. Bastos, J. M. C. De Portugal Martinez Vazquez, R. De Maria, M. Giovannozzi, M. Martino, and R. T. Garcia, Update of beam dynamics requirements for HL-LHC electrical circuits, CERN Technical Report No. CERN-ACC-2019-0030, 2019.

[47] Y. Papaphilippou, Detecting chaos in particle accelerators through the frequency map analysis method Chaos: An Interdisciplinary, J. Nonlinear Sci. 24, 024412 (2014).

[48] J. Laskar, Introduction to frequency map analysis, in Hamiltonian Systems with Three or More Degrees of Freedom (Springer, New York, 1999), pp. 134-150.

[49] J. Laskar, Frequency map analysis and particle accelerators, in Proceedings of the 2003 Particle Accelerator Conference, Vol. 1 (IEEE, New York, 2003), pp. 378-382.

[50] J. Laskar, Application of frequency map analysis, in The Chaotic Universe: Proceedings of the Second ICRA Network Workshop, Rome, Pescara, Italy, 1-5 February 1999 (World Scientific, Singapore, 2000), Vol. 10, p. 115.

[51] S. Kostoglou, N. Karastathis, Y. Papaphilippou, D. Pellegrini, and P. Zisopoulos, Development of computational tools for noise studies in the LHC, in Conf. Proc., IPAC-2017THPAB044 (2017), p. THPAB044, https://cds.cern.ch/ record/2289645/files/thpab044.pdf.
[52] J. Laskar, C. Froeschlé, and A. Celletti, The measure of chaos by the numerical analysis of the fundamental frequencies. application to the standard mapping, Physica D: Nonlinear Phenomena 56, 253 (1992).

[53] P. Zisopoulos, Y. Papaphilippou, and J. Laskar, Refined betatron tune measurements by mixing beam position data, Phys. Rev. Accel. Beams 22, 071002 (2019).

[54] T. Chen, A. Gerasimov, B. Cole, D. Finley, G. Goderre, M. Harrison, R. Johnson, I. Kourbanis, C. Manz, N. Merminga, L. Michelotti, S. Peggs, F. Pilat, S. Pruss, C. Saltmarsh, S. Saritepe, T. Satogata, R. Talman, C. G. Trahern, and G. Tsironis, Measurements of a Hamiltonian System and Their Description by a Diffusive Model, Phys. Rev. Lett. 68, 33 (1992).

[55] S. Papadopoulou, F. Antoniou, T. Argyropoulos, M. Fitterer, M. Hostettler, and Y. Papaphilippou, Modelling and measurements of bunch profiles at the LHC, J. Phys. Conf. Ser. 874, 012008 (2017).

[56] S. Papadopoulou, F. Antoniou, T. Argyropoulos, M. Hostettler, Y. Papaphilippou, and G. Trad, Impact of non-Gaussian beam profiles in the performance of hadron colliders, Phys. Rev. Accel. Beams 23, 101004 (2020).

[57] A. Romano, O. Boine-Frankenheim, X. Buffat, G. Iadarola, and G. Rumolo, Electron cloud buildup driving spontaneous vertical instabilities of stored beams in the Large Hadron Collider, Phys. Rev. Accel. Beams 21, 061002 (2018). 